\title{
Inaccuracy assessment for simultaneous measurement of resistivity and permittivity applying sensitivity and transfer function approaches
}

\author{
Alessandro Settimi ${ }^{1,},{\text { Achille } \text { Zirizzotti }^{1} \text {, James A. Baskaradas }}^{1}$, Cesidio Bianchi ${ }^{1}$ \\ ${ }^{1}$ Istituto Nazionale di Geofisica e Vulcanologia, sezione di Roma, Italy
}

\author{
Article history \\ Received October 18, 2009; accepted January 15, 2010. \\ Subject classification: \\ Instrumentation and techniques of general interest, Methods, Mathematical geophysics, Computational geophysics, Exploration geophysics.
}

\begin{abstract}
The present study proposes a theoretical modeling of simultaneous and noninvasive measurements of electrical resistivity and dielectric permittivity using a quadrupole probe on a subjacent medium. A mathematical-physical model is applied to the propagation of errors in the measurement of resistivity and permittivity based on a sensitivity functions tool. The findings are also compared with results of the classical method of analysis in the frequency domain, which is useful for determining the behavior of zero and pole frequencies in the linear time invariant circuit of the quadrupole. This study underlines that average values of electrical resistivity and dielectric permittivity can be used to estimate complex impedance over various terrains and concretes, especially when they are characterized by low levels of water saturation (content), and are analyzed within a bandwidth ranging only from low to middle frequencies. To meet the design specifications, that ensure satisfactory performances of the probe (inaccuracies of no more than 10\%), the forecasts provided by the sensitivity functions approach are discussed in comparison with those foreseen by the transfer functions method (in terms of both the band of frequency $\mathrm{f}$ and the measurable range of resistivity $\rho$, or permittivity $\varepsilon_{r}$ ).
\end{abstract}

\section{Introductory review}

\subsection{Electrical resistivity survey in soil science}

The electrical resistivity of a surface is a proxy for the spatial and temporal variability of many other physical properties of the subjacent medium. Samouellian [Samouëlian et al. 2005] discussed the basic principles of data interpretation and the main advantages and limits of such an analysis. This method allows nondestructive and very sensitive investigations, which can describe subsurface properties without direct inspection. Various techniques are applied according to the required scales of resolution and the heterogeneities of the area. A suitable probe injects generated electric currents into a medium, and the resulting potential differences are measured. The information is recovered from the potential-difference patterns, which provide the form of the medium heterogeneities and their electrical properties [Kearey et al. 2002]. The greater the electrical contrast between the subsurface matrix and a heterogeneity, the easier the detection. Other studies have shown that surface resistivity can be considered as a good indication of the variability of other physical properties [Banton et al. 1997]. The current pattern distributions depend on the medium heterogeneities and they are concentrated in a conductive volume. Some linear distributed arrays use four-electrode cells, which are commonly used in the laboratory for resistivity calibration [Rhoades et al. 1976] and in the field for vertical electrical sounding [Loke 2001].

\subsection{Middle frequency}

dielectric permittivity surveys in soil science

Analyses in the middle frequencies (MFs; $300 \mathrm{kHz}<f<3$ $\mathrm{MHz}$ ) allow measurements of dielectric permittivity. Fechant and Tabbagh [1999] developed an interesting approach, whereby they used a MF band for the characterization of permittivity in a natural media. This approach used an electrostatic quadrupole probe that was designed to measure resistivity at several hundreds of $\mathrm{kHz}$ [Tabbagh 1994]. A quadrupole working at a frequency $455 \mathrm{kHz}$ can measure permittivity for the determination of water content [Fechant 1996]. However, this approach requires calibration in the laboratory.

\subsection{Low frequency electrical resistivity}

and dielectric permittivity surveys in soil science

Analyses in the low frequencies (LFs; $30 \mathrm{kHz}<f<300$ $\mathrm{kHz}$ ) allow simultaneous measurements of both electrical resistivity and dielectric permittivity. A series of studies has shown that the resistivity and dielectric constant (the 


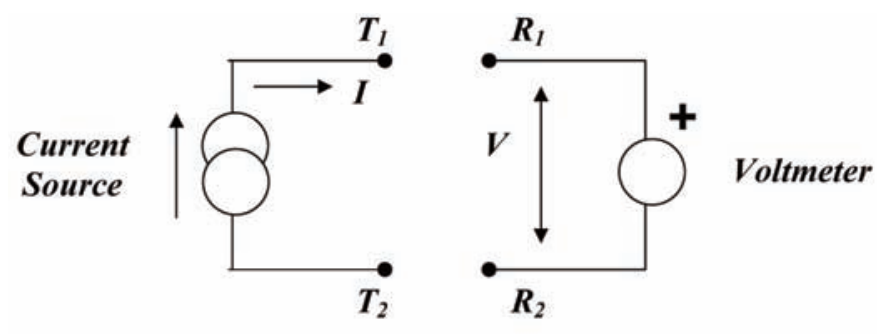

Figure 1. Equivalent circuit of the quadrupole probe.

complex permittivity) of a surface can be measured using a set of four electrodes [Grard 1990a, Grard 1990b, Grard and Tabbagh 1991, Tabbagh et al. 1993]. This novel approach was first introduced by Wenner [1915], and it improved on the system existing at the time, which provided only a resistivity assessment. In this new method, the four electrodes are manually inserted into the subjacent medium. Permittivity, which is sensitive to the presence of water, can also be determined using a LF probe (below $300 \mathrm{kHz}$ ) and it has an important role in the detection of anomalies in the subsurface.

Vannaroni and Del Vento [Vannaroni et al. 2004, Del Vento and Vannaroni 2005] used a dielectric spectroscopy probe to determine the complex permittivity of a surface from measurements of transfer impedance of a fourelectrode system that was electrically coupled to the medium. They defined the transfer impedance as the ratio between the voltage measured across a pair of receiving electrodes and the current transmitted by a second pair of electrodes [Vannaroni et al. 2004]. This impedance measurement was performed in an alternating current (AC) regime capacitive coupling, and it strongly depended on the geometry of the electrode array and also on the complex permittivity of the subsurface. The advantages offered by this method include that the exciter current can be injected into the surface even in the absence of galvanic contact, and that in the AC regime, both conduction and displacement currents of the medium can be measured, obtaining further information on the polarizability. In this case, the frequency band is $10 \mathrm{kHz}$ to $1 \mathrm{MHz}$, and the lower limit is effectively imposed by two aspects. First, the Maxwell-Wagner effect, which limits probe accuracy [Frölich 1990]. The most important limitation occurs because of interface polarization effects that are stronger at low frequencies, e.g. below $1 \mathrm{kHz}$, depending on the medium conductivity. Secondly, there is the need to maintain the amplitude of the current at measurable levels, as given the capacitive coupling between the electrodes and soil, the current magnitude is proportional to the frequency. On the other hand, the upper limit is opportunely fixed to allow analysis of the system in a regime of quasi static approximation and to neglect the velocity factor of the cables used for the electrode harness, which, in turn, reduces the accuracy of the mutual impedance phase measurements. Thus, it is possible to exploit the analysis of the system in the LF and MF bands where the electrostatic term is considerable. The general electromagnetic calculation provides lower values than the static case, and a high resistivity narrows the differences. So, in comparison, above $1 \mathrm{MHz}$ the general electromagnetic calculation must be preferred, while under $500 \mathrm{kHz}$ the static case would be used, and between $500 \mathrm{kHz}$ and $1 \mathrm{MHz}$ both of these methods can be used [Tabbagh et al. 1993].

The present study proposes a theoretical modeling of simultaneous and noninvasive measurements of electrical resistivity and dielectric permittivity using a quadrupole probe on a subjacent medium [see also arXiv.org's ref.: Settimi et al. 2009]. A mathematical-physical model is applied to the propagation of errors in the measurement of resistivity and permittivity based on the sensitivity functions tool. The findings are also compared with the results of the classical method of analysis in the frequency domain, which is useful for determining the behavior of zero and pole frequencies in the linear time invariant circuit of the quadrupole. This study underlines that average values of electrical resistivity and dielectric permittivity can be used to estimate complex impedance over various terrains and concretes, especially when they are characterized by low levels of water saturation or content [Knight and Nur 1987], and are analyzed within a frequency bandwidth only ranging from LFs to MFs [Al-Qadi et al. 1995, Myounghak et al. 2007]. To meet the design specifications that ensure the satisfactory performance of the probe (inaccuracies of no more than $10 \%$ ), the forecasts provided by the theory of error propagation suggested by Vannaroni et al. [2004] that apply the sensitivity functions approach, as explicitly developed in the study, are discussed in comparison to those foreseen by analysis in the frequency domain suggested by Grard and Tabbagh [1991]. Here, this deepens the transfer functions method to analyze the zero and pole behavior (in terms of both the band of frequency $f$ and the measurable range of resistivity $\rho$, or permittivity $\varepsilon_{r}$ ).

This study is organized as follows. Following this introductory review, Section 2 discusses the Cole-Cole empiric function. For simplicity of analysis, the dielectric dispersion is assumed to be very low, an operating condition that is satisfied when the electrical spectroscopy is performed only on nonsaturated water materials and especially in a suitable band of LFs and MFs. Section 3 introduces the quadrupole probe, and Section 4 provides the theoretical modeling that applies to both the sensitivity functions approach (Section 4.1.) and the transfer functions method (Section 4.2.). In Section 5, the configurations of the quadrupole are defined and discussed, and the conclusions are drawn up in Section 6. Finally, an outline of the somewhat lengthy calculations needed is presented in Appendices A and B. 


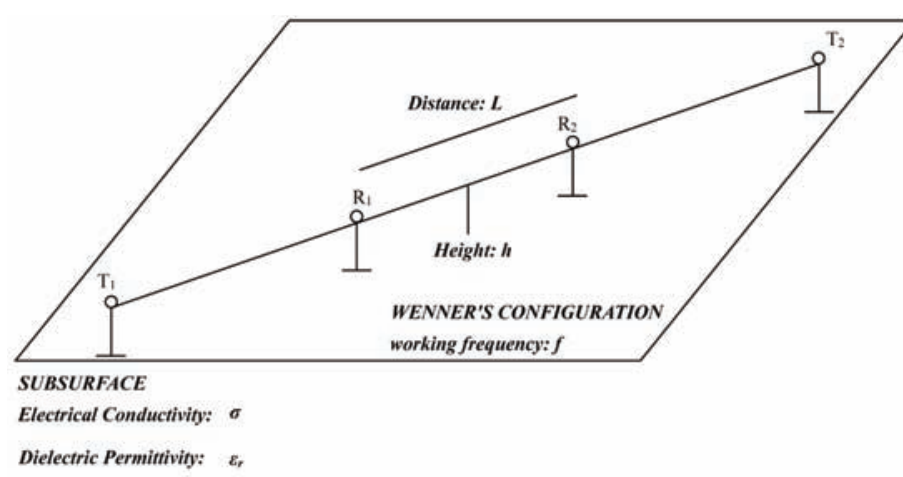

Figure 2a. Quadrupole probe in the linear Wenner configuration.

\section{Discussing Cole-Cole empiric functions}

According to Debye polarization mechanisms [Debye 1929] and Cole-Cole diagrams [Auty and Cole 1952], the complex permittivity of various materials in the frequency band from very LF to very high frequency (HF) shows several intensive relaxation effects and a non-trivial dependence on the water saturation [Chelidze and Gueguen 1999, Chelidze et al. 1999]. However, average values of electrical resistivity and dielectric permittivity can be used to estimate complex impedance over various terrains and concretes, especially when they are characterized by low water content [Knight and Nur 1987], and are analyzed within a frequency bandwidth ranging from only LFs to MFs [Al-Qadi et al. 1995, Myounghak et al. 2007].

Many functions have been proposed to fit dielectrics data. Among these, there are those obtained by attempts to model the physical processes, or those of simple empirical functions, which are used to parameterize the data without knowledge of the mechanisms involved. A widely used empirical function was proposed by the Cole brothers, and it is based on the theory of Debye relaxation, which was the first treatment of this phenomenon.

The Cole-Cole empiric function defines the first-order dielectric response of materials in the frequency domain,

$$
\begin{aligned}
& \varepsilon_{r}^{\text {complex }}(f)=\varepsilon_{r}(f)-j \frac{\sigma(f)}{2 \pi f \varepsilon_{0}}= \\
& =\varepsilon_{r, H}+\frac{\varepsilon_{r, L}-\varepsilon_{r, H}}{1+(j 2 \pi f \tau)^{1-\alpha}}-j \frac{\sigma_{L}}{2 \pi f \varepsilon_{0}},
\end{aligned}
$$

which consists of real and imaginary parts:

$$
\begin{aligned}
& \frac{\varepsilon_{r}(f)-\varepsilon_{r, H}}{\varepsilon_{r, L}-\varepsilon_{r, H}}=\frac{1+(2 \pi f \tau)^{1-\alpha} \sin (\alpha \pi / 2)}{1+(2 \pi f \tau)^{2(1-\alpha)}+2(2 \pi f \tau)^{1-\alpha} \sin (\alpha \pi / 2)}, \\
& \frac{\left[\sigma(f)-\sigma_{L}\right] / 2 \pi f \varepsilon_{0}}{\varepsilon_{r, L}-\varepsilon_{r, H}}= \\
& =\frac{(2 \pi f \tau)^{1-\alpha} \cos (\alpha \pi / 2)}{1+(2 \pi f \tau)^{2(1-\alpha)}+2(2 \pi f \tau)^{1-\alpha} \sin (\alpha \pi / 2)},
\end{aligned}
$$

where $\varepsilon_{0}$ is the dielectric constant in a vacuum.

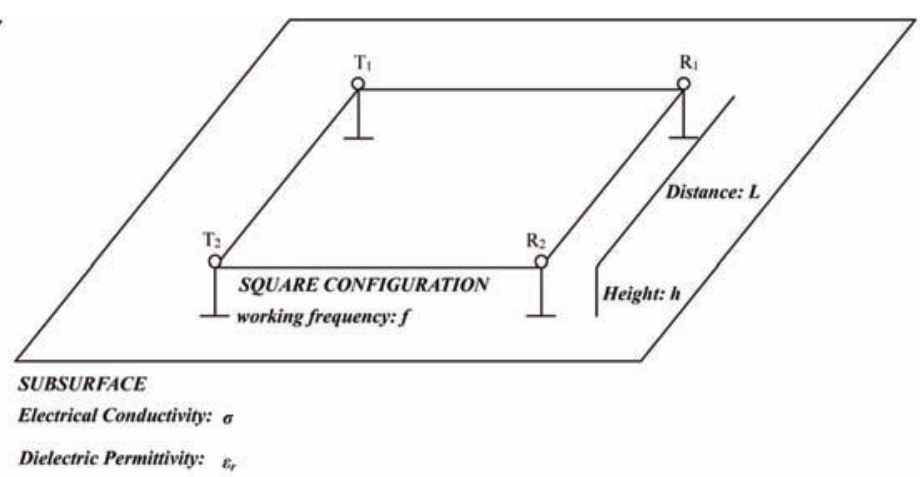

Figure $\mathbf{2 b}$. Quadrupole probe in the square configuration.

The electrical conductivity $\sigma(f)$ and dielectric permittivity $\varepsilon_{r}(f)$ show limited values at LFs and HFs, $\sigma_{L}, \varepsilon_{r, L}$ and $\sigma_{H}, \varepsilon_{r, H}$, which are linked by the relaxation time $\tau$ :

$$
\frac{\left(\varepsilon_{L}-\varepsilon_{H}\right) \varepsilon_{0}}{\tau}=\sigma_{H}-\sigma_{L},
$$

Thus, as can be seen, permittivity and conductivity cannot vary independently of each other [Frölich 1990].

At the characteristic frequency of relaxation $f_{c}=1 /(2 \pi \cdot \tau)$, the permittivity $\varepsilon_{r}$ assumes an intermediate value between the LFs and HFs, $\varepsilon_{r, L}$ and $\varepsilon_{r, H}$. Alternatively, the relaxation frequency $f_{c}$ can be considered as that frequency at which the conductivity $\sigma$ assumes the middle value between the two limiting values $\sigma_{L}$ and $\sigma_{H}$.

In reality, Equation (2.1) is a generalization of the Debye equation, which is designed to take into account the enlargement of the dispersion region due to the complexity of the structure and the composition of the materials, through the introduction of another parameter $\alpha$ (between 0 and 1). Note that for $\alpha=0$, Equation (2.1) can be reduced to exactly the Debye equation. It has to be underlined that the parameter $\alpha$ is an increasing function of the water saturation $S_{W}$, such that $\alpha\left(S_{W}=0\right) \rightarrow 0$, reaching a limiting value $\alpha_{L}>0$ for $S_{W} \rightarrow 1$ [Knight and Nur 1987]. Indeed, the complex dielectric permittivity is flattened with decreasing water content or increasing frequency [Al-Qadi et al. 1995, Myounghak et al. 2007].

The complex dielectric permittivity $\varepsilon_{r}^{\text {complex }}(f)$ can be approximated to a constant if its dominant term $\left(\varepsilon_{r, L}-\varepsilon_{r, H}\right) /$ $\left[1+(j 2 \pi f \tau)^{1-\alpha}\right]$ is a function that is almost independent of the frequency:

$$
(2 \pi f \tau)^{1-\alpha}<<1 .
$$

This operating condition of Equation (2.5) holds when the materials are characterized by low water content, i.e.:

$$
\alpha \rightarrow 0,
$$

and are analyzed over a band lower than the MFs, i.e.: 


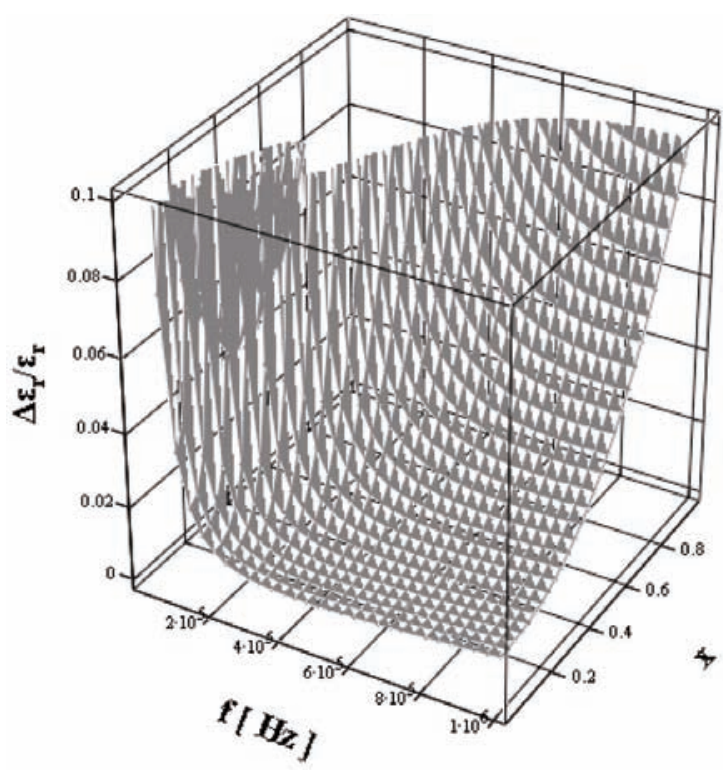

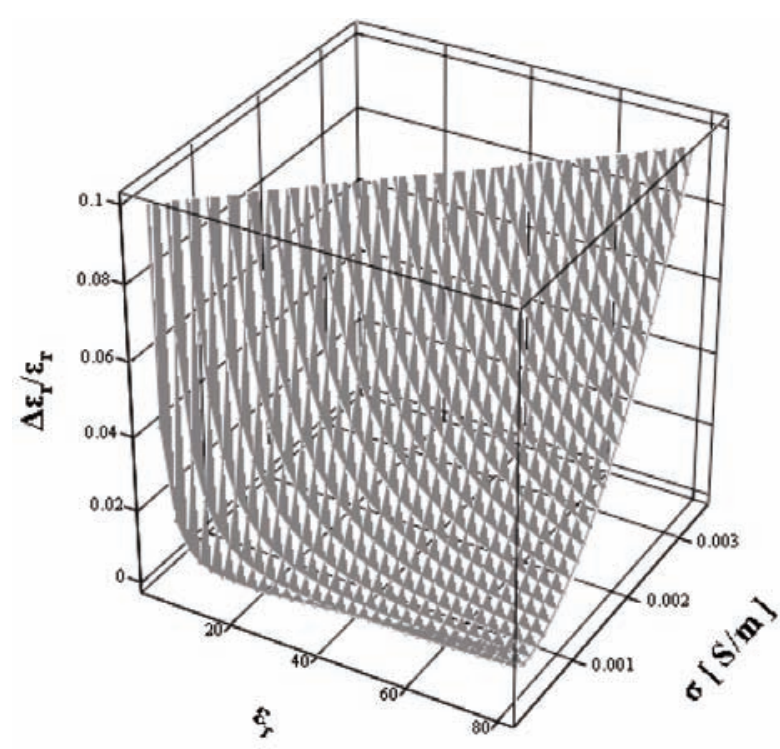

(a) (b)

Figure 3. On the hypothesis that $\Delta|Z| /|Z|=\Delta \Phi_{Z} / \Phi_{Z}=10^{-3}$, the inaccuracy $\Delta \varepsilon_{r} / \varepsilon_{r}$ in the measurement of the dielectric permittivity $\varepsilon_{r}$ plotted: (a) as a function $\Delta \varepsilon_{r} / \varepsilon_{r}(f, x)$ of both the frequency $f$ in the band $f \in\left[0, f_{\text {lim }}\right]$, with $f_{\text {lim }}=1 \mathrm{MHz}$, and the ratio $x=h / L$ between the height $h$ above ground and the characteristic geometrical dimension $L$, as $0<x \leq 1$, when the quadrupole probe designed in the linear Wenner configuration has a capacitive contact on a non-saturated concrete of low electrical conductivity, i.e. $\sigma=10^{-4} \mathrm{~S} / \mathrm{m}, \varepsilon_{r}=4$; (b) as a function $\Delta \varepsilon_{r} / \varepsilon_{r}\left(\sigma, \varepsilon_{r}\right)$ of both the conductivity $\sigma$ and the permittivity $\varepsilon_{r}$, when the quadrupole working in a fixed band $B=100 \mathrm{kHz}$ is in galvanic contact on a class of concretes such that $\sigma \in\left[10^{-4} \mathrm{~S} / \mathrm{m}, 2 \cdot 10^{-2} \mathrm{~S} / \mathrm{m}\right]$.

$$
f<\frac{1}{2 \pi \tau} .
$$

Indeed, the constant $\tau$ depends on the physical processes under consideration, and it has an order of magnitude that varies from a few picoseconds for the orientation of electrons and small dipolar molecules, up to a few seconds for the effects of counter-ions or for interfacial polarization [Frölich 1990].

Therefore, in the present study let us refer to the $\left(\sigma, \varepsilon_{r}\right)$ values in the LF to MF bandwidth proposed for various terrains by Edwards [1998] and for concretes by Polder et al. [2000] and Laurents et al. [2005].

\section{Quadrupole probe}

When using a quadrupole probe (Figure 1), the response depends on both geometrical parameters, like the height of each electrode above the ground surface and the separation of the electrodes, and physical parameters, including frequency, electrical conductivity and dielectric permittivity. When a medium is assumed to be linear and its response linearly dependent on the electrical charges of the two exciting electrodes, the simplest approach is a static calculation [Tabbagh et al. 1993], especially using a low operating frequency. If the electrodes have small dimensions relative to their separation, then they can be considered as points. Moreover, if the current wavelength is much larger than all of the dimensions under consideration, then the quasi-static approximation applies [Grard 1990a, Grard 1990b].

The quadrupole probe (Figure 1) measures a capacitance in a vacuum $C_{0}(L)$ that is directly proportional to its characteristic geometrical dimension, i.e. the electrodeelectrode distance $L$, both in a linear Wenner configuration (Figure 2a),

$$
C_{0}(L)=4 \pi \varepsilon_{0} \cdot L
$$

and in a square arrangement (Figure $2 b$ ),

$$
C_{0}(L)=\alpha \cdot 4 \pi \varepsilon_{0} L
$$

which is greater by a factor $\alpha=1 /\left(2-2^{1 / 2}\right)>1$, where $\varepsilon_{0}$ is the dielectric constant in a vacuum.

When the quadrupole specified by the electrodeelectrode distance $L$ has galvanic contact with the subjacent medium of electrical conductivity $\sigma$ and dielectric permittivity $\varepsilon_{r}$, it measures a transfer impedance $Z_{N}(f, L, \sigma$, $\varepsilon_{r}$ ) that consists of parallel components of resistance $R_{N}(L, \sigma)$ and capacitance $C_{N}\left(L, \varepsilon_{r}\right)$. The resistance $R_{N}(L, \sigma)$ depends only on $L$ and $\sigma$ [Grard and Tabbagh 1991]:

$$
R_{N}(L, \sigma)=2 \frac{\varepsilon_{0} / \sigma}{C_{0}(L)} ;
$$

while $C_{N}\left(L, \varepsilon_{r}\right)$ depends only on $L$ and $\varepsilon_{r}$ [Grard and Tabbagh 1991]:

$$
C_{N}\left(L, \varepsilon_{r}\right)=\frac{1}{2} C_{0}(L) \cdot\left(\varepsilon_{r}+1\right) .
$$

As a consequence, as well as grazing the medium, if the probe measures the conductivity $\sigma$ and permittivity $\varepsilon_{r}$ working in a frequency $f$ much lower than the cut-off frequency $f_{T}=f_{T}\left(\sigma, \varepsilon_{r}\right)=\sigma /\left(2 \pi \varepsilon_{0}\left(\varepsilon_{r}+1\right)\right)$, the transfer 


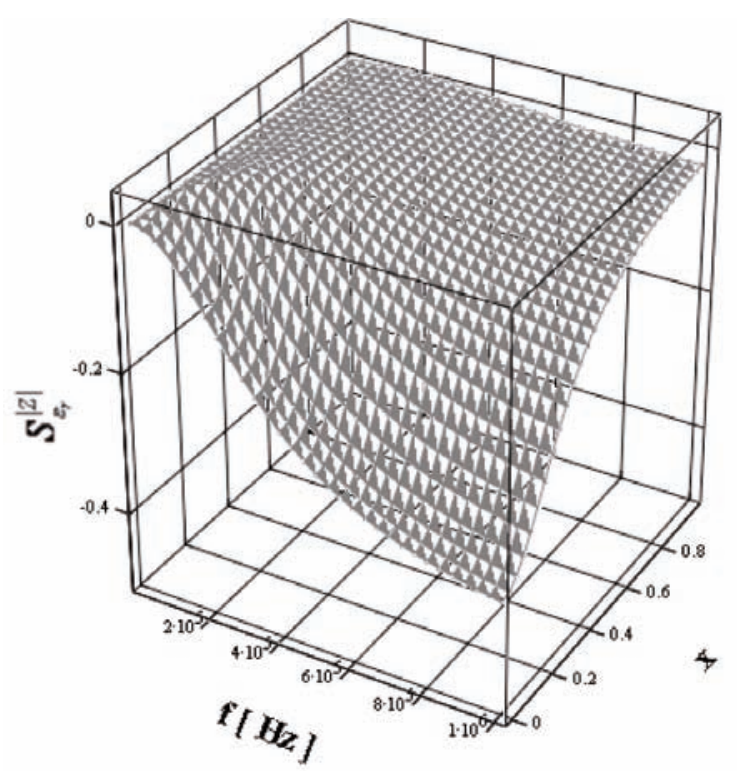

(a)

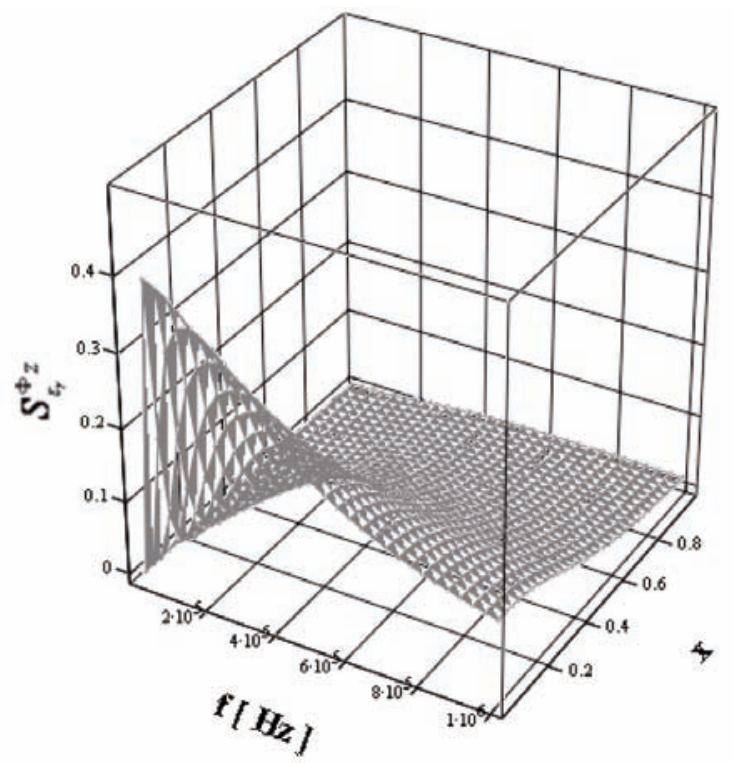

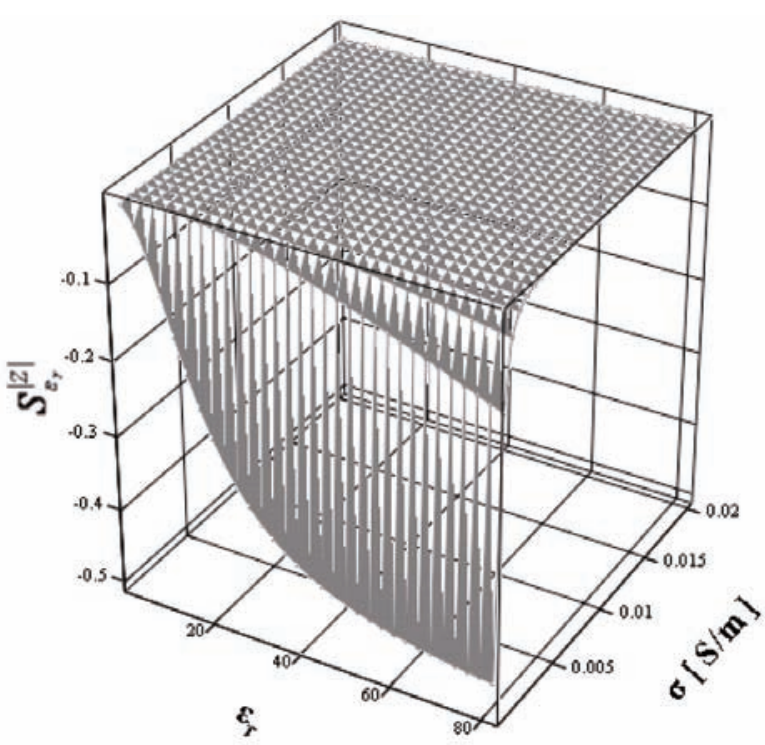

(b)

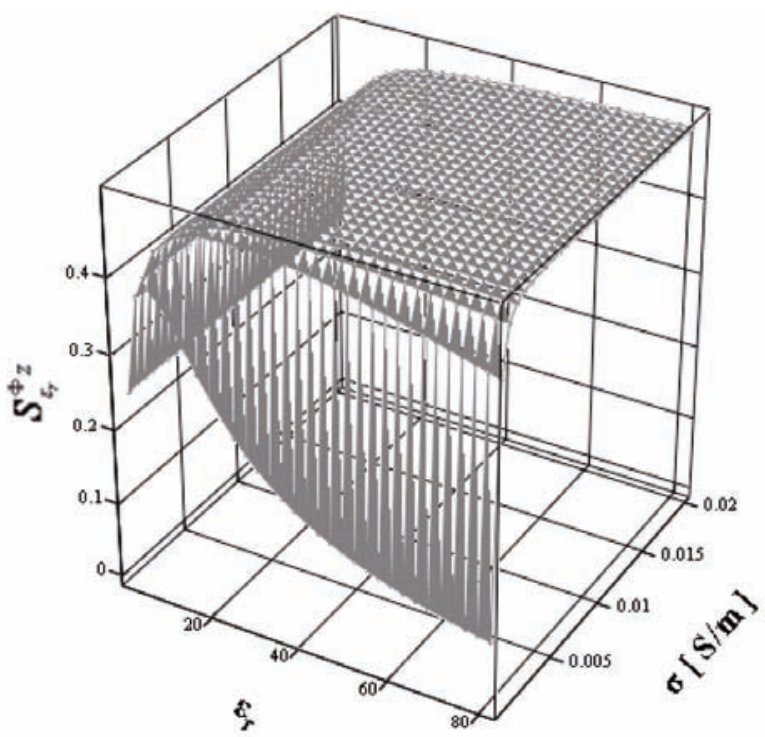

(d)

Figure 4. Sensitivity functions $S_{\varepsilon_{r}}^{|Z|}$ and $S_{\varepsilon_{t}}^{\Phi_{z}}$ for the transfer impedance both in modulus $|Z|$ and in phase $\Phi_{Z}$, relative to the dielectric permittivity $\varepsilon_{r}$, plotted: (a, c) as functions $S_{\varepsilon_{e}}^{|z|}(f, x)$ (a) and $S_{\varepsilon_{z}}^{\phi_{z}}(f, x)$ (c) of both the working frequency $f$ and the height/dimension ratio $x=h / L$ under the same operative conditions as Figure $3 \mathrm{a} ;(\mathrm{b}, \mathrm{d})$ as functions $S_{\varepsilon_{r}}^{|z|}(\sigma, \varepsilon)(\mathrm{b})$ and $S_{\varepsilon_{r}}^{\phi_{z}}(\sigma, \varepsilon)(\mathrm{d})$ of both the conductivity $\sigma$ and the permittivity $\varepsilon_{r}$ under the same operative conditions as Figure $3 \mathrm{~b}$.

impedance $Z_{N}\left(f, L, \sigma, \varepsilon_{r}\right)$ is characterized by the phase $\Phi_{N}(f$, $\left.\sigma, \varepsilon_{r}\right)$ and modulus $|Z|_{N}(L, \sigma)$. The phase $\Phi_{N}\left(f, \sigma, \varepsilon_{r}\right)$ depends linearly on $f$, with a maximum value of $\pi / 4$, and it is directly proportional to the ratio $\left(\varepsilon_{r}+1\right) / \sigma$; while $|Z|_{N}(L$, $\sigma$ ) does not depend on $f$, and is inversely proportional to both $L$ and $\sigma$. Indeed, if $Z_{N}\left(f, L, \sigma, \varepsilon_{r}\right)$ consists of the parallel components of $R_{N}(L, \sigma)$ - see Equation (3.3) - and $C_{N}\left(L, \varepsilon_{r}\right)$ - see Equation (3.4) -, then it is fully characterized by the HF pole $f_{T}=f_{T}\left(\sigma, \varepsilon_{r}\right)$, which cancels its denominator: the transfer impedance acts as a LF-MF band-pass filter with cut-off $f_{T}=$ $f_{T}\left(\sigma, \varepsilon_{r}\right)$; in other words, the frequency equalizing Joule and displacement current. Under the operating conditions defined in Section 2, average values of $\sigma$ can be used over the band ranging from $L F$ to MF; therefore, $|Z|_{N}(L, \sigma)$ is not a function of frequency below $f_{T}$.
Instead, when the quadrupole probe (Figure 1) has capacitive contact with the subjacent medium and the geometry of the probe is characterized by the ratio $x$ between the height above ground $h$ and the electrodeelectrode distance $L$,

$$
x=\frac{h}{L},
$$

its configurations can be entirely defined by a suitable geometrical factor $K(x)$, which depends on the height/ dimension ratio $x$. This was introduced by Grard and Tabbagh [1991], and can be specified for the linear Wenner configuration (Figure 2a):

$$
K(x)=2\left(1+4 x^{2}\right)^{-1 / 2}-\left(1+x^{2}\right)^{-1 / 2},
$$




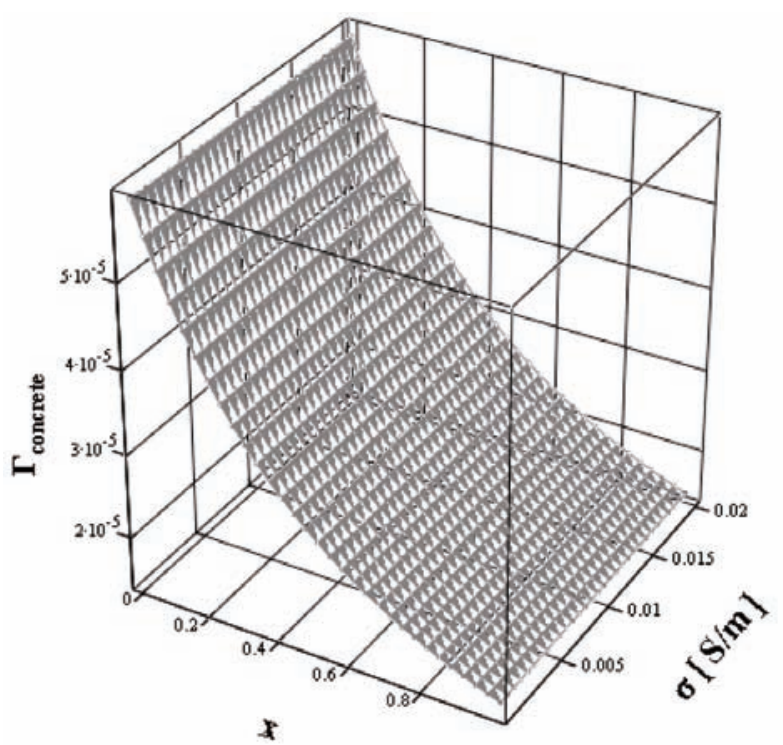

Figure 5. Ratio $\Gamma=\Gamma_{1} / \Gamma_{2}$ between the first member $\Gamma_{1}$ and the second member $\Gamma_{2}$ of Equation (B.8), plotted as a function $\Gamma(x, \sigma)$ of both the height/dimension ratio $x=h / L$ and the electrical conductivity $\sigma$, with the quadrupole probe designed in the linear Wenner configuration and in capacitive contact on a selected concrete of dielectric permittivity $\varepsilon_{r}=4$.

and the square arrangement (Figure $2 b$ ):

$$
K(x)=\frac{\left(1+4 x^{2}\right)^{-1 / 2}-2^{-1 / 2}\left(1+2 x^{2}\right)^{-1 / 2}}{1-2^{-1 / 2}} .
$$

Actually, Grard and Tabbagh [1991] preferred to introduce the complementary $\delta(x)$ of the geometrical factor $K(x)$, i.e.:

$$
\delta(x)=1-K(x)
$$

where $K(x=0)=1$ and $\delta(x=0)=0$.

So, if the quadrupole works in the pulse frequency $\omega=2 \pi f$, which can be normalized with respect to the cut-off $\omega_{T}=2 \pi f_{T}$ [Grard and Tabbagh 1991],

$$
\Omega=\frac{\omega}{\omega_{T}}=\omega R_{N} C_{N}=\omega \frac{\varepsilon_{0}\left(\varepsilon_{r}+1\right)}{\sigma},
$$

then the probe measures a transfer impedance $Z\left(\Omega, x, \sigma, \varepsilon_{r}\right)$ which consists of the resistance $R\left(\Omega, x, \sigma, \varepsilon_{r}\right)$ and capacitance $C\left(\Omega, x, \sigma, \varepsilon_{r}\right)$ parallel components [Grard and Tabbagh 1991],

$$
\begin{aligned}
& R\left(\Omega, x, \sigma, \varepsilon_{r}\right)= \\
& =R_{N}(L, \sigma) \frac{\left[1+\delta(x) \frac{\varepsilon_{r}-1}{2}\right]^{2}+\left[\frac{\delta(x)}{\Omega} \frac{\varepsilon_{r}+1}{2}\right]^{2}}{1-\delta(x)}, \\
& C\left(\Omega, x, \sigma, \varepsilon_{r}\right)= \\
& =C_{N}\left(L, \varepsilon_{r}\right) \frac{1+\delta(x)\left(\frac{\varepsilon_{r}-1}{2}+\frac{1}{\Omega^{2}} \frac{\varepsilon_{r}+1}{2}\right)}{\left[1+\delta(x) \frac{\varepsilon_{r}-1}{2}\right]^{2}+\left[\frac{\delta(x)}{\Omega} \frac{\varepsilon_{r}+1}{2}\right]^{2}} .
\end{aligned}
$$

Inverting Equations (3.10) and (3.11), $\sigma$ and $\varepsilon_{r}$ can be expressed as functions of $R$ and $C$, i.e.:

$$
\sigma(\omega, x, R, C)=\frac{2[1-\delta(x)] \varepsilon_{0} \omega^{2} R C_{0}}{\delta^{2}(x)+\omega^{2} R^{2}\left[C_{0}-\delta(x) C\right]^{2}},
$$

$$
\begin{aligned}
& \varepsilon_{r}(\omega, x, R, C)= \\
& \frac{\delta(x)[\delta(x)-2]-\omega^{2} R^{2}\left[C_{0}-\delta(x) C\right]\left\{C_{0}+[\delta(x)-2] C\right\}}{\delta^{2}(x)+\omega^{2} R^{2}\left[C_{0}-\delta(x) C\right]^{2}} .
\end{aligned}
$$

In our opinion, once the degrees of freedom of the $(f, x)$ pair are fixed, it is not suitable to choose $(R, C)$ as independent variables and then $\left(\sigma, \varepsilon_{r}\right)$ as dependent variables (Equations 3.12 and 3.13). Instead, it is more appropriate to consider ( $\sigma$, $\left.\varepsilon_{r}\right)$ as quantities of physical interest, and consequently Equations (3.10) and (3.11) as the starting points for the theoretical development. Indeed, even if the physics does not forbid the choice of $(R, C)$ as independent variables, applying the function $(R, C) \rightarrow\left(\sigma, \varepsilon_{r}\right)$, the procedures of the design should anyway choose $\left(\sigma, \varepsilon_{r}\right)$ as independent variables, preferentially applying the inverse function $\left(\sigma, \varepsilon_{r}\right) \rightarrow(R, C)$. According to the following two practical approaches: (a) $\left(\sigma, \varepsilon_{r}\right)$ as independent variables in order - to establish the class of media with conductivity and permittivity $\left(\sigma, \varepsilon_{r}\right)$ that can be investigated by a quadrupole working in a fixed band $\mathrm{B}$ and specified by a known geometry $\mathrm{x}$; (b) preferential way $\left(\sigma, \varepsilon_{r}\right) \rightarrow(R, C)$ since - once a subjacent medium with electrical conductivity $\sigma$ and dielectric permittivity $\varepsilon_{r}$ is selected, the quadrupole probe specifications $R$ and $C$ can be projected both in frequency $f$ and in height/dimension ratio $x$.

\section{Theoretical modeling}

The measurements taken using the quadrupole probe are affected by errors that mainly originate from uncertainties associated with transfer impedance, from dishomogeneities between the modeled and the actual stratigraphy, and from inaccuracies of the electrode array deployment above the surface [Vannaroni et al. 2004]. Errors in impedance result mainly from uncertainties in the electronic systems that perform the amplitude and phase measurements of the voltages and currents [Del Vento and Vannaroni 2005]. These uncertainties were assumed to be constant throughout the whole frequency band, even though their effects that propagate through the transfer function will produce a frequency-dependent perturbation.

\subsection{Sensitivity functions approach}

This study proposes to develop explicitly the sensitivity functions approach that is implied in the theory of error 


\section{APPROACH BY THE SENSITIVITY FUNCTIONS}
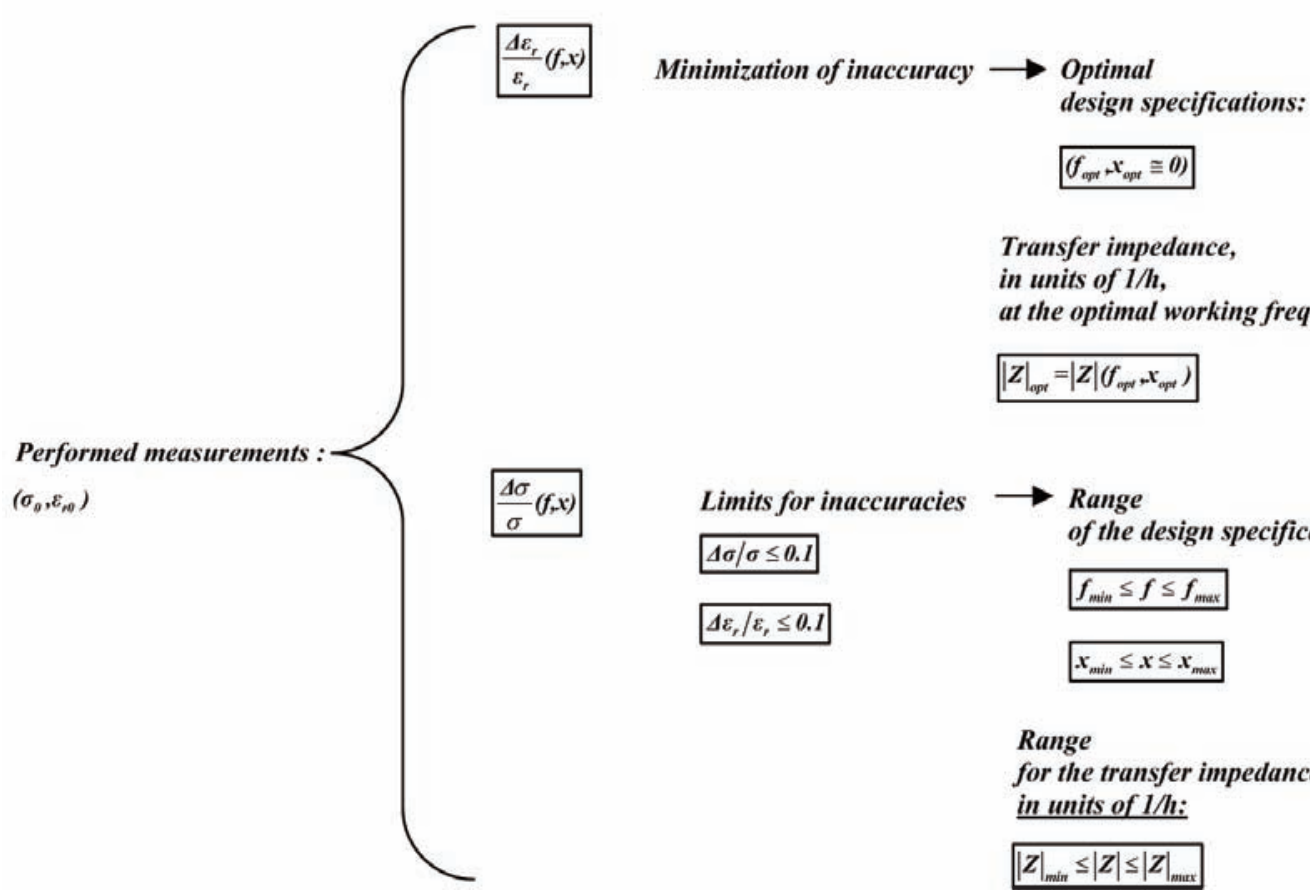

Transfer impedance,

in units of $1 / \mathrm{h}$,

at the optimal working frequency:

$|Z|_{\text {epr }}=|Z| \sigma_{\text {ovt }}, x_{\text {opt }} \mid$

Limits for inaccuracies

$\longrightarrow$ Range

of the design specifications:

$\Delta \sigma / \sigma \leq 0.1$

$\Delta \varepsilon_{r} / \varepsilon_{r} \leq 0.1$

$f_{\min } \leq f \leq f_{\max }$

$x_{\min } \leq x \leq x_{\max }$

Range

for the transfer impedance,

in units of $1 / \mathrm{h}$ :

$|Z|_{\min } \leq|Z| \leq|Z|_{\max }$

TRANSFER FUNCTION METHOD
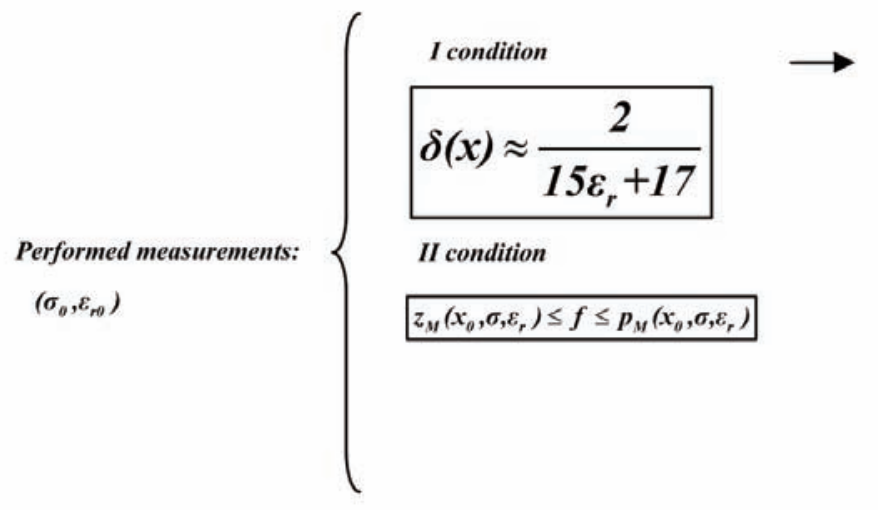

Ratio height/dimension:

$x_{\text {spt }}^{\prime}$

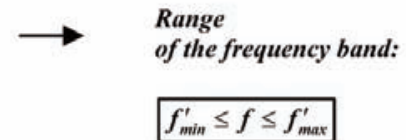

Range

for the transfer impedance,

in units of $1 / \mathrm{h}$ :

$|Z|_{\min }^{\prime} \leq|Z| \leq|Z|_{\max }^{\prime}$

Figure 6. Conceptual schemes for numerical simulations to design the characteristic geometrical dimensions and the frequency band, limiting inaccuracies in the measurements of the quadrupole probe in capacitive contact with selected materials as non-saturated concretes, in the hypothesis that $\Delta|Z| /|Z|=\Delta \Phi_{Z} / \Phi_{Z}=10^{-3}$.

propagation suggested by Vannaroni et al. [2004]. Indeed, this section introduces a mathematical-physical model for the propagation of errors in the measurement of electrical conductivity $\sigma$ and dielectric permittivity $\varepsilon_{r}$, based on the sensitivity functions tool [Murray-Smith 1987]. This is useful for expressing inaccuracies in the measurements of conductivity and permittivity (Figure 3) as a linear combination of the inaccuracies for the transfer impedance, both in modulus $|Z|$ and in phase $\Phi_{Z}$, where the weight functions are inversely proportional only to the sensitivity functions for $|Z|$ and $\Phi_{Z}$ relative to $\sigma$ and $\varepsilon_{r}$ (Figure 4). The inaccuracies of transfer impedance depend on the inaccuracies of electrical voltage and current that are assigned by the electronics used, and in particular, by the sampling methods.

Therefore, the inaccuracies $\Delta \sigma / \sigma$ in the measurement of the electrical conductivity $\sigma$, and $\Delta \varepsilon_{r} / \varepsilon_{r}$ in the dielectric permittivity $\varepsilon_{r}$, can be expressed as a linear combination of the inaccuracies $\Delta|Z| /|Z|$ and $\Delta \Phi_{Z} / \Phi_{Z}$ in the measurement of the transfer impedance, respectively in modulus $|Z|$ and in phase $\Phi_{Z}$,

$$
\begin{aligned}
& \frac{\Delta \sigma}{\sigma}=\left|S_{|Z|}^{\sigma}\right| \frac{\Delta|Z|}{|Z|}+\left|S_{\Phi_{Z}}^{\sigma}\right| \frac{\Delta \Phi_{Z}}{\Phi_{Z}}= \\
& =\frac{1}{\left|S_{\sigma}^{|Z|}\right|} \frac{\Delta|Z|}{|Z|}+\frac{1}{\left|S_{\sigma}^{\Phi_{z}}\right|} \frac{\Delta \Phi_{Z}}{\Phi_{Z}} \text {, for } \varepsilon_{r}=\text { const, }
\end{aligned}
$$


APPROACH BY THE SENSITIVITY

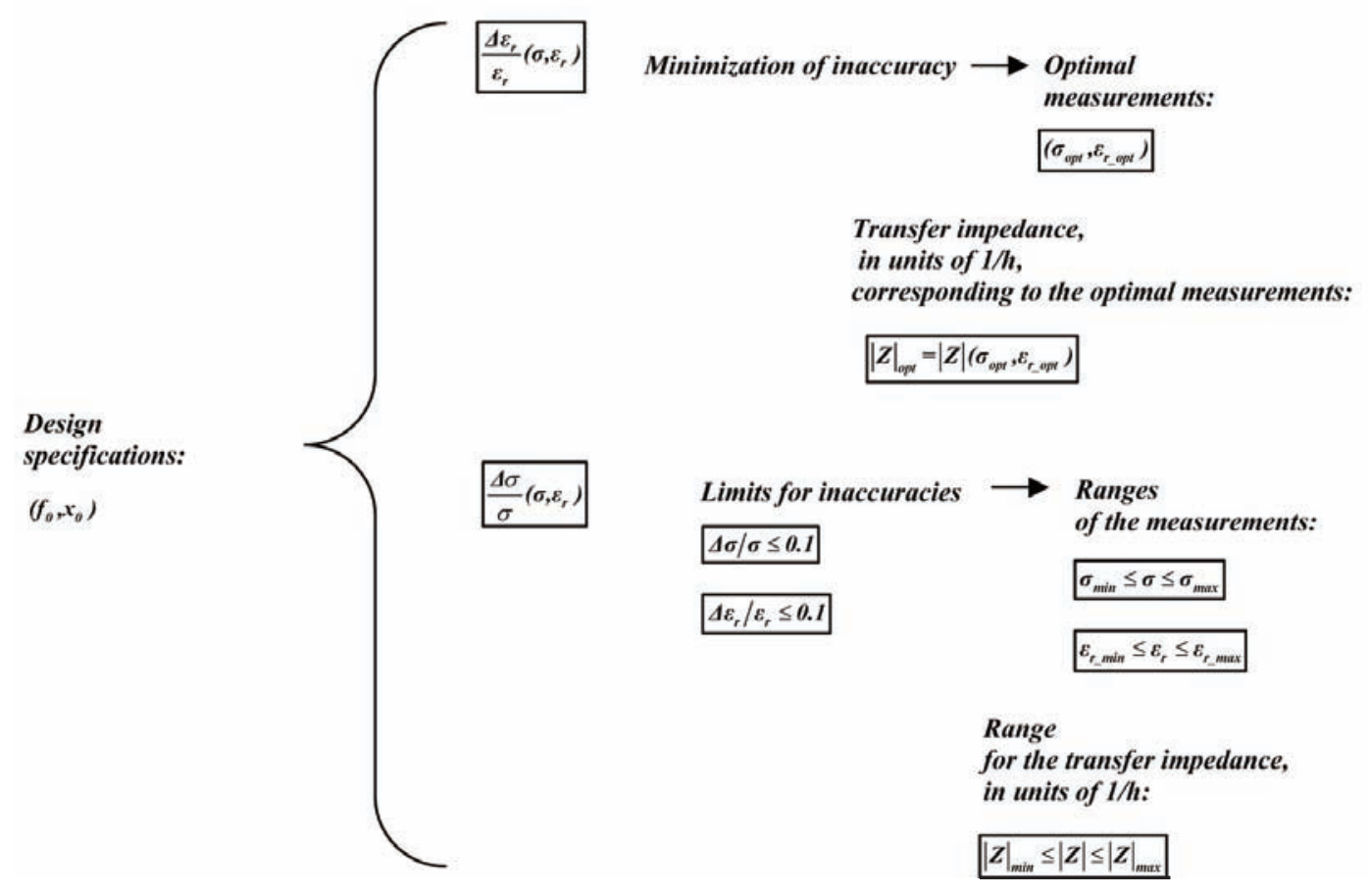

TRANSFER FUNCTION METHOD
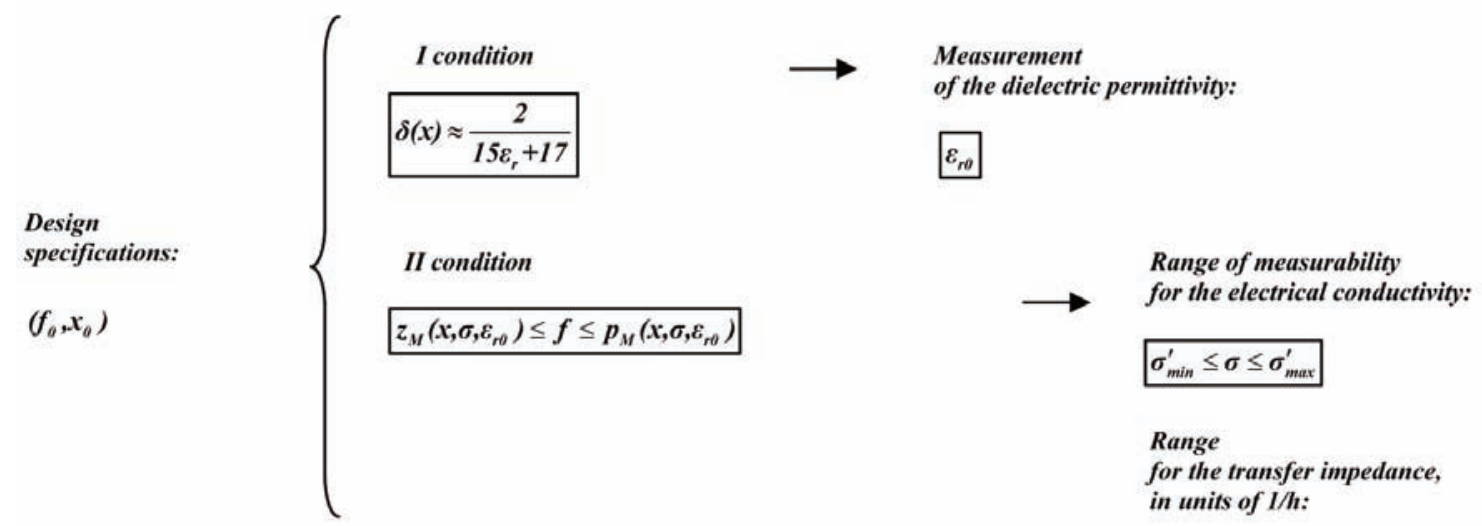

$|Z|_{\min }^{\prime} \leq|Z| \leq|Z|_{\max }^{\prime}$

Figure 7. Conceptual schemes for numerical simulations to establish the measurable ranges of electrical conductivity and dielectric permittivity, limiting inaccuracies in the measurements of the quadrupole probe in capacitive contact, and fixing its optimum working frequencies and characteristic geometrical dimensions $\left[\Delta|Z| /|Z|=\Delta \Phi_{Z} / \Phi_{Z}=10^{-3}\right]$.

$$
\begin{aligned}
& \frac{\Delta \varepsilon_{r}}{\varepsilon_{r}}=\left|S_{|Z|}^{\varepsilon_{r}}\right| \frac{\Delta|Z|}{|Z|}+\left|S_{\Phi_{z}}^{\varepsilon_{r}}\right| \frac{\Delta \Phi_{Z}}{\Phi_{Z}}= \\
& =\frac{1}{\left|S_{\varepsilon_{r}}^{|Z|}\right|} \frac{\Delta|Z|}{|Z|}+\frac{1}{\left|S_{\varepsilon_{r}}^{\Phi_{2}}\right|} \frac{\Delta \Phi_{Z}}{\Phi_{Z}}, \text { for } \sigma=\mathrm{const},
\end{aligned}
$$

where $\left(S_{\sigma}^{|Z|}, S_{\sigma}^{\Phi_{z}}\right)$ and $\left(S_{\varepsilon_{r}}^{|Z|}, S_{\varepsilon_{r}}^{\Phi_{z}}\right)$ are the pairs of sensitivity functions for the transfer impedance, both in $|Z|$ and $\Phi_{Z}$, relative to the conductivity $\sigma$ and permittivity $\varepsilon_{r}$, the expressions for which are reported in Appendix A. The conditions $\sigma=$ const and $\varepsilon_{r}=$ const in Equations (4.1) and (4.2) underline not so much that constant values of electrical conductivity and dielectric permittivity are used to estimate the complex impedance over various terrains and concretes under the operating conditions defined in section 2 , but that the quantities $\sigma$ and $\varepsilon_{r}$ are not independent of each other, since the electrical displacement shows a phase-shift with respect to the electrical field [Frölich 1990]. So, for the need to distinguish the inaccuracies in measurements of conductivity and permittivity, the inaccuracy $\Delta \sigma / \sigma$ can only be calculated assuming there is no uncertainty for $\varepsilon_{r}\left(\Delta \varepsilon_{r} / \varepsilon_{r}\right.$ $=0 \leftrightarrow \varepsilon_{r}=$ const), and vice versa.

Moreover, according to the physical problem, the probe performs measurements of the transfer impedance $Z$, both in modulus $|Z|$ and in phase $\Phi_{Z}$, which are characterized by the inaccuracies $\Delta|Z| /|Z|>0$ and $\Delta \Phi_{Z} / \Phi_{Z}>0$. Mathematically, application of the conditions $|Z|=$ const or $\Phi_{Z}=$ const is not 

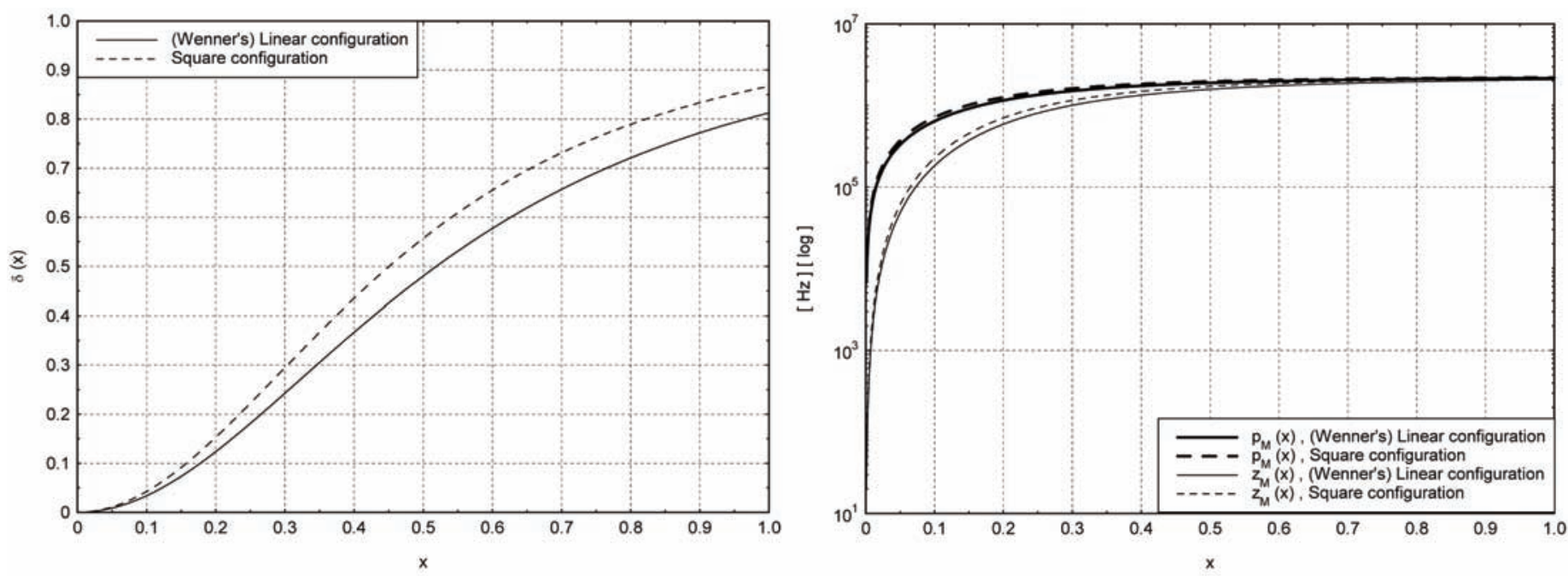

(a)

(b)
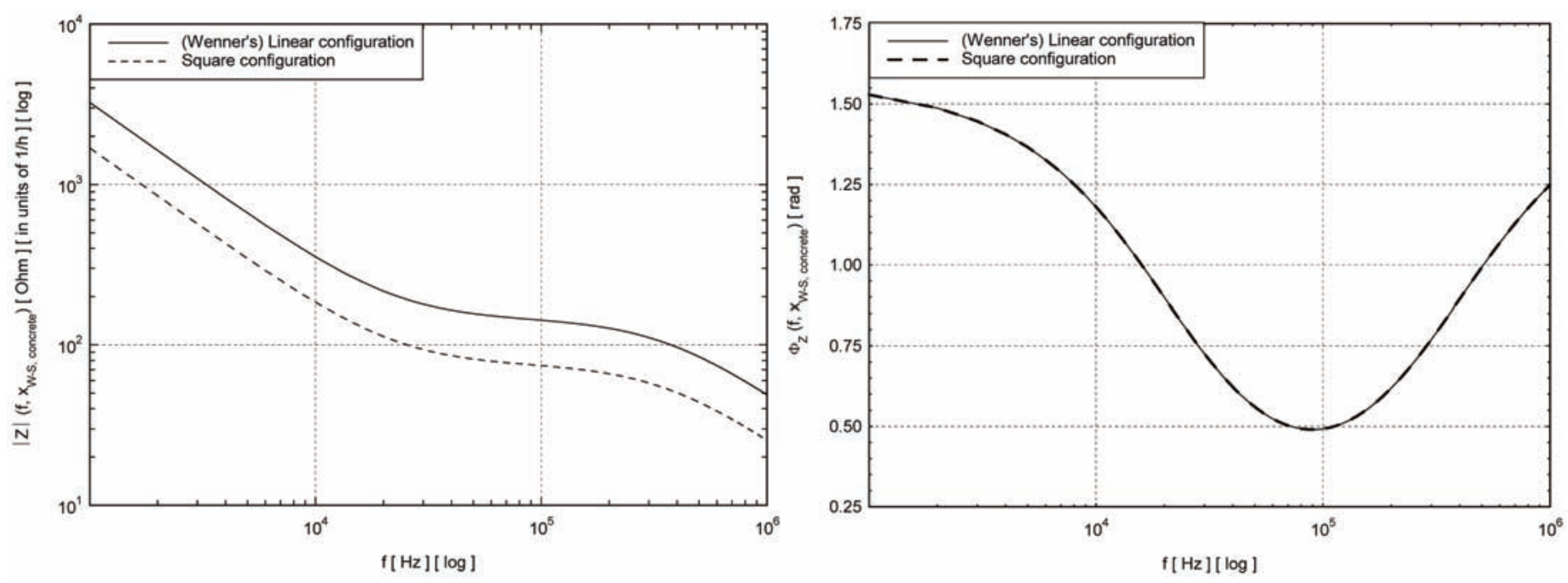

(c)

Figure 8. Referring to a quadrupole probe designed in the linear Wenner or square configurations and with capacitive contact on a non-saturated concrete of low electrical conductivity, i.e. $\sigma=10^{-4} \mathrm{~S} / \mathrm{m}, \varepsilon_{r}=4$ : (a) plots, as a function of the ratio $x=h / L$ between the height $h$ above ground and the characteristic geometrical dimension $L$, with $0<x \leq 1$, for the geometrical factor $\delta(x)$; (b) semi-logarithmic plots for both the zero $z_{M}(x)$ and pole $p_{M}(x)$ of the transfer impedance in modulus; (c, d) Bode's diagrams, as a function of the frequency $f$ in the band $f \in\left[0, f_{\text {lim }}\right]$, with $f_{\text {lim }}=1 \mathrm{MHz}$, for the transfer impedance both in modulus $|Z|\left(f, x_{\text {concrete }}\right)$, units of $1 / \mathrm{h}$, and phase $\Delta \Phi_{Z}\left(f, x_{\text {concretet }}\right) ;(\mathrm{e})$ - see next page - on the hypothesis that $\Delta|Z| /|Z|=\Delta \Phi_{Z} / \Phi_{Z}=10^{-3}$, semi-logarithmic plots for both the inaccuracies $\Delta \varepsilon_{r} / \varepsilon_{r}\left(f, x_{\text {concrete }}\right)$ in the measurement of the permittivity $\varepsilon_{r}$, and $\Delta \sigma / \sigma\left(f, x_{\text {concrete }}\right)$ of the conductivity $\sigma$, with the height $/$ dimension ratio designed optimally in the linear Wenner $\left(x_{W, \text { concrete }}=0.087\right)$ and square $\left(x_{S, \text { concrete }}=0.078\right)$ configurations.

allowed. In this context, the sensitivity functions $S_{|Z|}^{\sigma}$ and $S_{|Z|}^{\varepsilon_{r}}$ cannot be calculated assuming $\Phi_{Z}=$ const, and then the sensitivities $S_{\Phi_{Z}}^{\sigma}$ and $S_{\Phi_{z}}^{\mathcal{E}_{r}}$ assuming $|Z|=$ const. Indeed, as discussed above, once the degrees of freedom of the $(f, x)$ pair are fixed, it is not suitable to choose $\left(|Z|, \Phi_{Z}\right)$ or $(R, C)$ as independent variables. Consequently, the sensitivity functions cannot be calculated by the dependent variables $\sigma$ $=\sigma\left(|Z|, \Phi_{Z}\right)$ and $\varepsilon_{r}=\varepsilon_{r}\left(|Z|, \Phi_{Z}\right)$ or by Equations (3.12) and (3.13). Instead, the physical problem should be approached recalling that $\left(f, x, \sigma, \varepsilon_{r}\right)$ have been considered as independent variables. In the simplifying hypothesis that the frequency $f$ and the height/ dimension ratio $x$ are characterized by inaccuracies $\Delta f / f \approx 0$ and $\Delta x / x \approx 0$ close to zero, the conditions $f=$ const and $x=$ const can be applied. Necessarily, the inaccuracy $\Delta \sigma / \sigma$ in the measurement of the electrical conductivity $\sigma$ is calculated assuming $\varepsilon_{r}=$ const, and then the inaccuracy $\Delta \varepsilon_{r} / \varepsilon_{r}$ for the dielectric permittivity $\varepsilon_{r}$ is calculated assuming $\sigma=$ const. As a consequence, the mathematical calculations should be done recalling that Equations (3.10) and (3.11) have been considered as the starting points for the theoretical development. The inaccuracies $\Delta \sigma / \sigma$ for the conductivity $\sigma$ and $\Delta \varepsilon_{r} / \varepsilon_{r}$ for the permittivity $\varepsilon_{r}$ can be more directly expressed as functions of $\left(f, x, \sigma, \varepsilon_{r}\right)$ by calculating the sensitivity functions $\left(S_{\sigma}^{|Z|}, S_{\sigma}^{\Phi_{z}}\right)$ and $\left(S_{\varepsilon_{r}}^{|Z|}, S_{\varepsilon_{r}}^{\Phi_{z}}\right)$ in the last part of Equations (4.1) and (4.2). These sensitivities are derived from the transfer impedance $1 / Z=1 / R+j \omega C$ reported in Equations (3.10) and (3.11).

The interesting physical results obtained using this sensitivity functions approach are discussed below. If the quadrupole probe is in galvanic contact with the subsuface, 


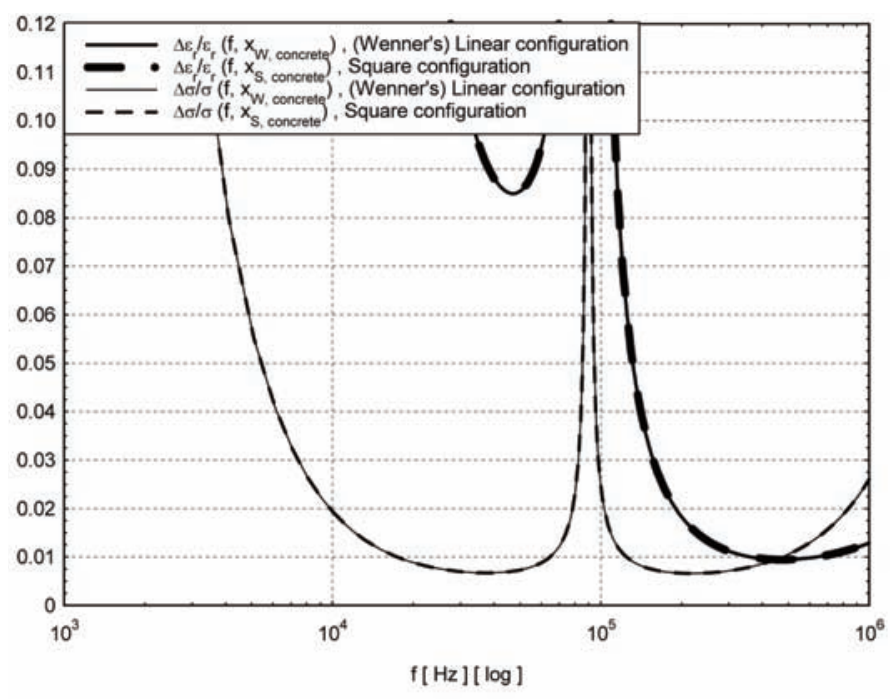

(e)

Figure 8e. Caption on previous page.

i.e. $h=0$, then the inaccuracies $\Delta \sigma / \sigma$ in the measurement of the electrical conductivity $\sigma$, and $\Delta \varepsilon_{r} / \varepsilon_{r}$ for the dielectric permittivity $\varepsilon_{r}$, are minimized in the frequency band $B$ of the quadrupole, for all of its geometrical configurations and media, and even if $h \neq 0$, the design of the probe must still be optimized with respect to the minimum value of the inaccuracy $\Delta \varepsilon_{r} / \varepsilon_{r}$ for $\varepsilon_{r}$, which is always higher than the corresponding minimum value of the inaccuracy $\Delta \sigma / \sigma$ in the band $B$ of the probe, for all of its configurations and media [Tabbagh et al. 1993, Vannaroni et al. 2004].

Under the quasi static approximation, only if the quadrupole probe is in galvanic contact with the subjacent medium, i.e. $h=0$, and considering that the sensitivities functions are defined as normalized functions, then our mathematical-physical model predicts that the sensitivities of the transfer impedance relative to the conductivity $\sigma$ and permittivity $\varepsilon_{r}$ are independent of the characteristic geometrical dimension of the quadrupole, i.e. the electrodeelectrode distance $\mathrm{L}$.

If the probe grazes the medium, then the transfer impedance $Z_{N}(\sigma, L)$ consists of the resistance $R_{N}(\sigma, L)$, which is independent of $\varepsilon_{r}$, and the parallel capacitance $C_{N}$ $\left(\varepsilon_{r}, L\right)$, which is independent of $\sigma$, such that: the sensitivity function $S_{\sigma}^{R}$ for $R$ relative to $\sigma$ is a constant equal to $(-1)$; the sensitivity $S_{\varepsilon_{r}}^{C}\left(\varepsilon_{r}\right)$ for $C$ relative to $\varepsilon_{r}$ is independent of $\sigma$, and behaves as the function $\varepsilon_{r} /\left(\varepsilon_{r}+1\right)$ of $\varepsilon_{r}$; the $S_{\varepsilon_{r}}^{R}$ function for $R$ relative to $\varepsilon_{r}$ and the $S_{\sigma}^{C}$ function for $C$ relative to $\sigma$ are identically zero. As a consequence, the inaccuracy $\Delta R / R$ for $R$ shows the same behavior versus the frequency of the inaccuracy $\Delta \sigma / \sigma$ in the measurement of $\sigma$, as $\Delta R / R=\left|S_{\sigma}^{R}\right|$ $\Delta \sigma / \sigma=\Delta \sigma / \sigma$, and the inaccuracy $\Delta C / C$ for $C$ shows a similar behavior versus the frequency with respect to the inaccuracy $\Delta \varepsilon_{r} / \varepsilon_{r}$ for $\varepsilon_{r}$, as $\Delta C / C=\left|S_{\varepsilon_{r}}^{C}\left(\varepsilon_{r}\right)\right| \Delta \varepsilon_{r} / \varepsilon_{r} \approx \Delta \varepsilon_{r} / \varepsilon_{r}$ if $\varepsilon_{r}>>1$. Moreover, as well as the hypothesis $h=0$, if $\sigma$ and $\varepsilon_{r}$ are measured in the cut-off frequency $f_{T}=f_{T}\left(\sigma, \varepsilon_{r}\right)$, then: the sensitivity functions $S_{\sigma}^{|Z|}$ and $S_{\sigma}^{\Phi_{z}}$ for the transfer impedance, both in modulus $|Z|$ and in phase $\Phi_{Z}$, relative to $\sigma$, are constant, and respectively $(-1 / 4)$ and $(-1 / \pi)$; and the sensitivities $S_{\mathcal{E}_{r}}^{|Z|}\left(\varepsilon_{r}\right)$ and $S_{\varepsilon_{r}}^{\Phi_{z}}\left(\varepsilon_{r}\right)$ for $|Z|$ and $\Phi_{Z}$ relative to $\varepsilon_{r}$ are independent of $\sigma$, such that they behave as the function $\varepsilon_{r} /\left(\varepsilon_{r}+1\right)$ of $\varepsilon_{r}$. As a consequence, the ratio between $\Delta \varepsilon_{r} / \varepsilon_{r}$ and $\Delta \sigma / \sigma$ is independent of $\sigma$, and behaves as the function $\left(1+1 / \varepsilon_{r}\right)$ of $\varepsilon_{r}$, and $\Delta \sigma / \sigma$ is a constant equal to $\Delta \sigma / \sigma=$ $4 \Delta|Z| /|Z|+\pi \Delta \Phi_{Z} / \Phi_{Z}$. As a post-test, only assuming the conditions $\sigma=$ const and $\varepsilon_{r}=$ const in Equations (4.1) and (4.2), the sensitivity functions approach provides results that are in agreement with a previous report [Vannaroni et al. 2004].

\subsection{Transfer functions method}

This study proposes to deepen the transfer functions method by analyzing the zero and pole behavior, which were implied in the frequency domain analysis suggested by Grard and Tabbagh [1991]. Indeed, this section introduces the method of analysis in the frequency domain for determining the behavior of the zero and pole frequencies in the linear time-invariant circuit of the quadrupole probe (Figure 1). To satisfy the operative conditions of linearity for the measurements, if the quadrupole has capacitive contact with the subjacent medium then the frequency $f$ of the probe should be imposed as included between the zero $z_{M}$ and the pole $p_{M}$ of the transfer impedance, and so its modulus is almost constant within the frequency band [Grard and Tabbagh 1991],

$$
z_{M}\left(x, \varepsilon_{r}, \sigma\right) \leq f \leq p_{M}\left(x, \varepsilon_{r}, \sigma\right) .
$$

Based on the above conditions, an optimization equation is deduced for the probe that links the optimal ratio $x$ between its height above ground and its characteristic geometrical dimension only to the dielectric permittivity $\varepsilon_{r}$ of the medium, so that:

$$
\delta(x) \approx \frac{2}{15 \varepsilon_{r}+17} .
$$

To satisfy the operative conditions of linearity for the measurements, if the quadrupole is in galvanic contact with the subjacent medium, then the working frequency $f$ of the quadrupole should be imposed as lower than the cut-off frequency of the transfer impedance, and so its modulus as constant below the cut-off frequency. It is only under these conditions that it is optimal to design the characteristic geometrical dimensions of the probe or to establish the measurable ranges of the conductivity $\sigma$ and permittivity $\varepsilon_{r}$ of the medium (Figure 5). Equations (4.3) and (4.4) that were derived by the classical transfer function method are demonstrated in Appendix B.

The interesting physical results obtained using this transfer functions method are discussed below. To meet the 


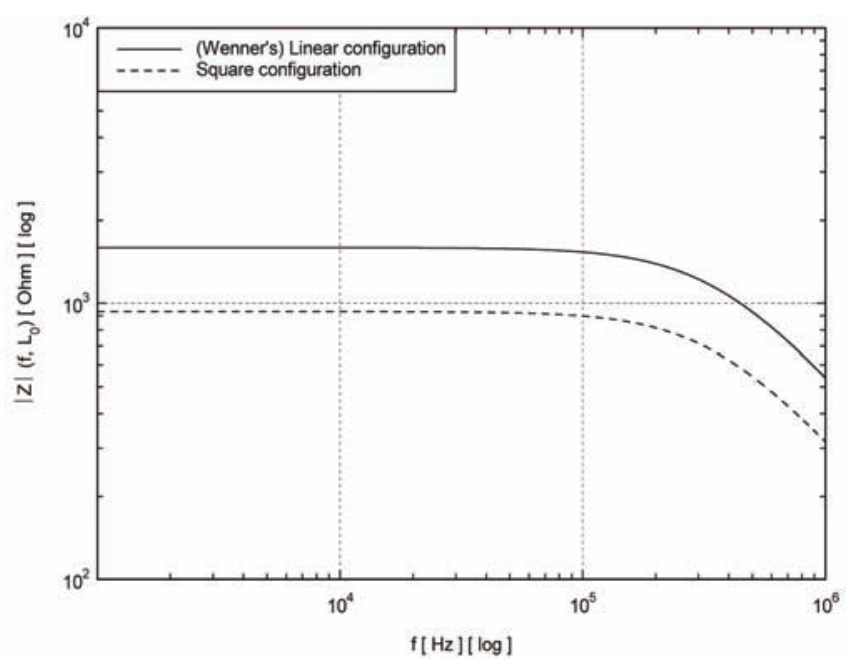

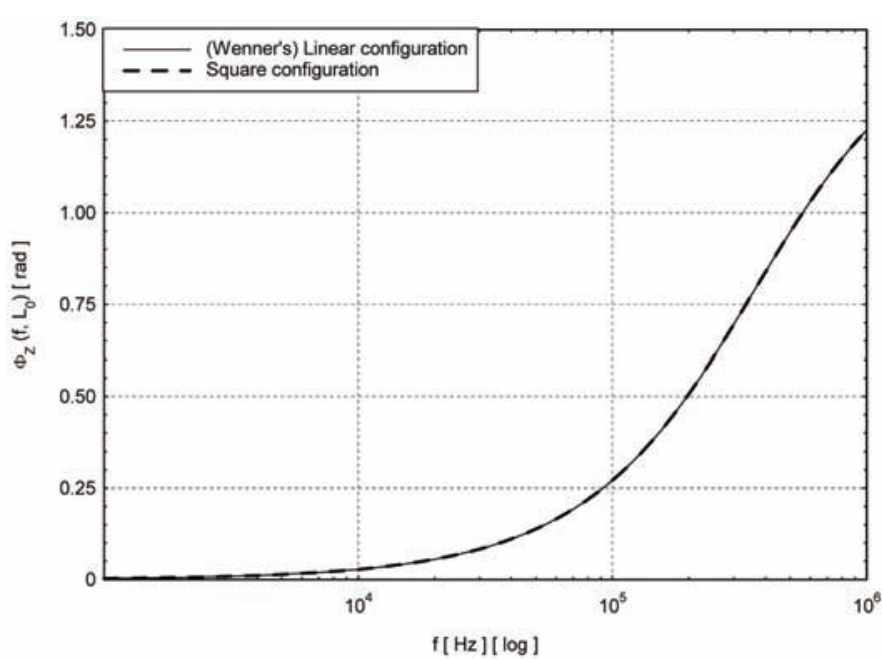

(b)

(a)

Figure 9. With reference to a quadrupole probe designed according to an electrode-electrode distance $L_{0}=1 \mathrm{~m}$ and in galvanic contact on a concrete of low electrical conductivity, i.e. $\sigma=10^{-4} \mathrm{~S} / \mathrm{m}, \varepsilon_{r}=4$ : (a, b) Bode's diagrams, as functions of the frequency $f$ for the transfer impedance both in modulus $|Z|\left(f, L_{0}\right)\left(\right.$ a) and phase $\Phi_{Z}\left(f, L_{0}\right)$ (b); (c) semi-logarithmic plots for both the inaccuracies $\Delta \sigma / \sigma(f)$ in the measurement of the conductivity $\sigma$, and $\Delta \varepsilon_{r} / \varepsilon_{r}(f)$ of the permittivity $\varepsilon_{r}\left[\Delta|Z| /|Z|=\Delta \Phi_{Z} / \Phi_{Z}=10^{-3}\right]$.

design specifications that ensure satisfactory performances of the probe (inaccuracy of no more than $10 \%$ ), the forecasts provided by the theory of error propagation suggested by Vannaroni et al. [2004] that apply the sensitivity functions approach, as explicitly developed in the study, are less stringent than those foreseen by the analysis in the frequency domain suggested by Grard and Tabbagh [1991]. Here, this deepens the transfer function method to analyze the zero and pole behavior, in terms of both larger band of frequency $f$ and wider measurable range of resistivity $\rho$ or permittivity $\varepsilon_{r}$ (Figures 6, 7).

Indeed, given a surface (e.g. a non-saturated concrete with low conductivity $\sigma=10^{-4} \mathrm{~S} / \mathrm{m}$ and $\varepsilon_{r}=4$ ) with dielectric permittivity $\varepsilon_{r}$ (Figure 6):

if the quadrupole probe has capacitive contact with the subjacent medium, i.e. $h \neq 0$, then having defined an optimal ratio $x_{\text {opt }}=h_{\text {opt }} / L$ between an optimal height hopt above ground and the characteristic geometrical dimension $L$, the transfer impedance $Z\left(f, x_{\text {opt }}\right)$, in units of $1 / h_{\text {opt }}$, calculated in $x_{o p t}$, is a function of the working frequency $f$ such that its modulus $|Z|\left(f, x_{\text {opt }}\right)$, in units of $1 / h_{\text {opt }}$, is almost constant between a zero frequency $z\left(x_{\text {opt }}\right)$ almost one LF decade higher than a minimum frequency value $f_{\text {min }}\left(x_{\text {opt }}\right)$, allowing the inaccuracy $\Delta \varepsilon_{r} / \varepsilon_{r}\left(f, x_{\text {opt }}\right)$ in the measurement of $\varepsilon_{r}$ below a prefixed limit (10\%), and a pole $p\left(x_{\text {opt }}\right)$ almost one MF decade lower than the maximum value of frequency $f_{\max }\left(x_{\text {opt }}\right)$ that satisfies the requirement that the inaccuracy $\Delta \varepsilon_{r} / \varepsilon_{r}\left(f, x_{o p t}\right)$ for $\varepsilon_{r}$ below $10 \%$ (Figure 8 );

if $h=0$, i.e. the quadrupole of the electrode-electrode distance $L$ grazes a medium of conductivity $\sigma$, then the transfer impedance $Z(f, L)$, calculated in $L$ is a function of the working frequency $f$ such that its modulus $|Z|(f, L)$ is constant down to the cut-off frequency $f_{T}=f_{T}\left(\sigma, \varepsilon_{r}\right)$, which is higher than an optimal frequency $f_{\text {opt }}(L)$ that minimizes the inaccuracy $\Delta \varepsilon_{r} / \varepsilon_{r}(f, L)$. Materials characterized by a low $\sigma$ or a high $\varepsilon_{r}$ lead to a leftward shift of the cut-off frequency $f_{T}$, so reducing the optimal frequency $f_{\text {opt }}(L)$ (Figure 9);

on a selected surface, it is usually possible to verify that the probe in capacitive contact performs optimal measurements over the band $\left[f_{\min }\left(x_{o p t}\right)<z\left(x_{o p t}\right), f_{\max }\left(x_{o p t}\right)>p\left(x_{o p t}\right)\right]$, which is shifted towards lower and higher frequencies compared to when the probe is in galvanic contact, where the respective band $\left(f_{\text {min }}, f_{\text {max }}\right)$ is narrower by almost one LFMF decade in frequency, particularly increasing the value of $\varepsilon_{r}$ (Figures $8 \mathrm{e}, 9 \mathrm{c}$ ).

Moreover, once the frequency band $B$ is fixed (Figure 7): if the quadrupole probe has capacitive contact with the

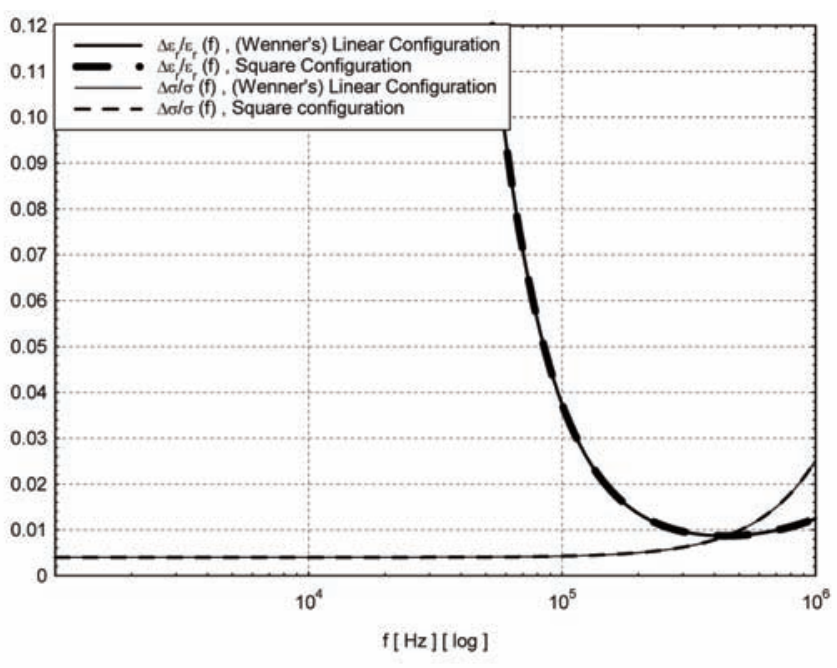

(c)

Figure 9c. 
Permittivity

inaccuracy

(a)

\begin{tabular}{ll}
\hline$x_{W, o p t}$ & $1.083 \cdot 10^{-4}$ \\
$\varepsilon_{r, o p t}$ & 6.703 \\
$\sigma_{\text {opt }}$ & $3.52 \cdot 10^{-5} \mathrm{~S} / \mathrm{m}$
\end{tabular}

$\varepsilon_{r, \text { opt }}=6.70$

$\Delta \varepsilon_{r} / \varepsilon_{r} \leq 0.1$

$\sigma_{\text {opt }}=3.52 \cdot 10^{-5} \mathrm{~S} / \mathrm{m}$

$\Delta \sigma / \sigma \leq 0.1$

(b)

$x_{W, \text { low }} \quad \approx 0$

$x_{W, \text { up }}$

0.475

$x_{W, \text { opt }}=1.083 \cdot 10^{-4}$

$\Delta \varepsilon_{r} / \varepsilon_{r} \leq 0.1$

$\Delta \sigma / \sigma \leq 0.1$

(c)

$\varepsilon_{\text {r, low }}, \sigma_{\text {low }}$

$1,5.333 \cdot 10^{-5} \mathrm{~S} / \mathrm{m}$

$\varepsilon_{r, u p}, \sigma_{u p}$

$81,3.14 \cdot 10^{-3} \mathrm{~S} / \mathrm{m}$

Table 1. Wenner's configuration: (a) optimal point $\left(x_{W, o p t}, \varepsilon_{r, \text { opp }}, \sigma_{\text {opt }}\right)$ of permittivity inaccuracy; (b) range of $x$ where $\Delta \varepsilon_{r} / \varepsilon_{r} \leq 0.1$ and $\Delta \sigma / \sigma \leq 0.1$, once selected optimally $\varepsilon_{r \text {, opt }}$ and $\sigma_{\text {opt }}$; (c) domains of $\varepsilon$ and $\sigma$ where $\Delta \varepsilon_{r} / \varepsilon_{r} \leq 0.1$ and $\Delta \sigma / \sigma \leq 0.1$, once fixed optimally $x_{W, o p t}$.

Domain $\left(\varepsilon_{\mathrm{r}}, \sigma\right)$ for $\Delta \varepsilon_{\mathrm{r}} / \varepsilon_{\mathrm{r}}\left(\mathrm{x}_{\text {concrete }}\right) \leq 0.1$

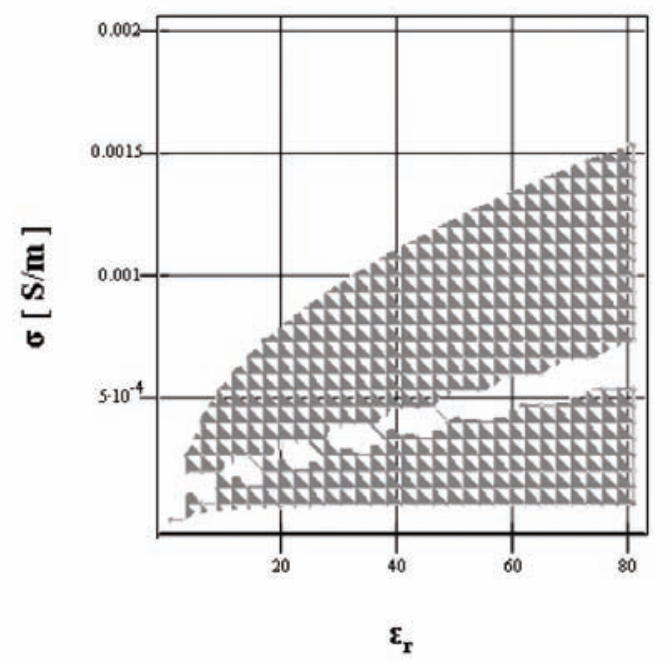

subjacent medium, then the ratio $x=h / L$ between the height $h$ above ground and the characteristic geometrical dimension $L$ ranges from the lower limit $x_{\text {low }}$, corresponding to water $\left(\varepsilon_{r}=81\right)$. In a preliminary analysis based on the transfer functions approach, it follows that the quadrupole designed with the height / dimension ratio $x=h / L$ optimally measures the dielectric permittivity $\varepsilon_{r, \text { opt }}$; the modulus $|Z|(x$, $\left.\sigma, \varepsilon_{r, \text { opt }}\right)$, in units of $1 / h$, of its transfer impedance, calculated in $x$ and $\varepsilon_{r, o p t}$, is a function of the electrical conductivity $\sigma$, is characterized by a zero $z\left(\sigma, \varepsilon_{r, \text { opt }}\right)$ and a pole $p\left(\sigma, \varepsilon_{r, \text { opt }}\right)$ frequency, which fall near the lower and upper limits of $B$, respectively, when $\sigma$ is measured within the range of the lower limit $\sigma_{\text {low }}^{\prime}$ and the upper limit $\sigma_{\text {up }}^{\prime}$. In a deeper analysis based on the sensitivity functions method, and still designing the quadrupole with the ratio $x=h / L$ for optimal measurement of $\varepsilon_{r, \text { opt }}$, it is possible to verify the measurable range of $\sigma$; the inaccuracy $\Delta \varepsilon_{r} / \varepsilon_{r}\left(x, \sigma, \varepsilon_{r, \text { opt }}\right)$ in the measurement of $\varepsilon_{r, \text { opt }}$, a function of $\sigma$, is below a prefixed limit $(10 \%)$ if $\sigma$ is measured within the range $\left(\sigma_{\text {low }}, \sigma_{\text {up }}\right)$, larger than $\left(\sigma_{\text {low }}^{\prime}, \sigma_{\text {up }}^{\prime}\right)$ by almost one order of magnitude, both the right and left sides (Figure 10, Tables 1,2);

if $h=0$, i.e. the probe of the electrode-electrode distance $L$ grazes a medium of conductivity $\sigma$ and permittivity $\varepsilon_{r}$, then the transfer impedance $Z\left(L, \sigma, \varepsilon_{r}\right)$ calculated in $L$ is a function of $\sigma$ and $\varepsilon_{r}$ such that its cut-off frequency $f_{T}=f_{T}\left(\sigma, \varepsilon_{r}\right)$, a function of both $\sigma$ and $\varepsilon_{r}$, ranges from $f_{T, \text { min }}=100 \mathrm{kHz}$ to $f_{\text {T,max }}=1 \mathrm{MHz}$ for materials belonging to a $\left(\sigma, \varepsilon_{r}\right)$-domain, which is almost superimposable with the corresponding one
Domain $\left(\varepsilon_{\mathrm{r}}, \sigma\right)$ for $\Delta \sigma / \sigma\left(\mathrm{x}_{\text {concrete }}\right) \leq 0.1$

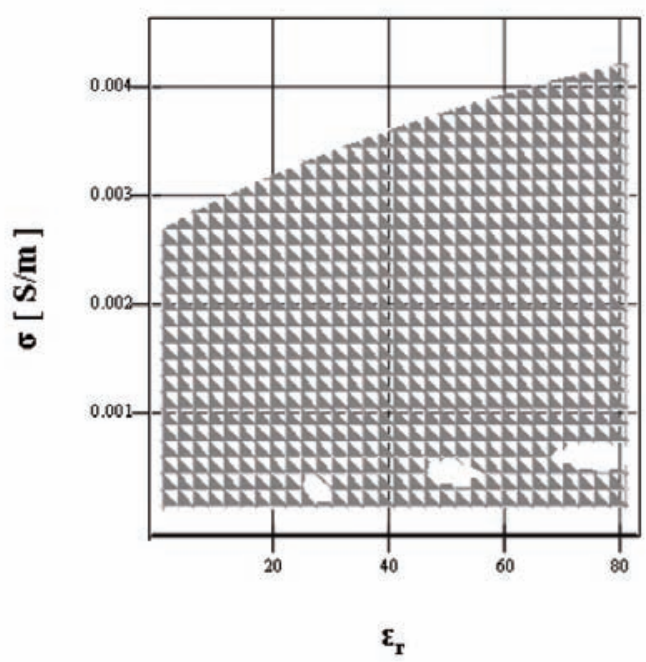

(a)

Figure 10. On the hypothesis that $\Delta|Z| /|Z|=\Delta \Phi_{Z} / \Phi_{Z}=10^{-3}$, referring to both the inaccuracies $\Delta \sigma / \sigma\left(\sigma, \varepsilon_{r}\right)$ for the electrical conductivity $\sigma$, and $\Delta \varepsilon_{r} / \varepsilon_{r}\left(\sigma, \varepsilon_{r}\right)$ for the dielectric permittivity $\varepsilon_{r}$, as functions of $\sigma$ and $\varepsilon_{r}$, and when the quadrupole probe is designed in the linear Wenner configuration working in a fixed band $B=100 \mathrm{kHz}$, with an height/dimension ratio $x_{W, \text { concrete }}=0.087$ which is optimal for capacitive contact only with a non-saturated concrete of permittivity $\varepsilon_{r}=4$ : (a, b) as plots for the orthogonal projections over the $\left(\sigma, \varepsilon_{r}\right)$ plane that satisfy the conditions $\Delta \sigma / \sigma\left(\sigma, \varepsilon_{r}\right) \leq 0.1(\mathrm{a})$ and $\Delta \varepsilon_{r} / \varepsilon_{r}\left(\sigma, \varepsilon_{r}\right) \leq 0.1(\mathrm{~b})$. See also Tables 1 and 2 . 


\begin{tabular}{lcc}
$\varepsilon_{r, \text { concrete }}=4.026$ & Sensitivity function approach & Transfer function method \\
$x_{W, \text { concrete }}=0.087$ & $4.473 \cdot 10^{-6} \mathrm{~S} / \mathrm{m}$ & $1.78 \cdot 10^{-5} \mathrm{~S} / \mathrm{m}$ \\
\hline$\sigma_{\text {low }}$ & $3.058 \cdot 10^{-4} \mathrm{~S} / \mathrm{m}$ & $7.12 \cdot 10^{-5} \mathrm{~S} / \mathrm{m}$ \\
$\sigma_{\text {up }}$ &
\end{tabular}

(a)

$$
\begin{array}{lc}
x_{W, \text { concrete }}=0.087 & \begin{array}{c}
\Delta \varepsilon_{r} / \varepsilon_{r} \leq 0.1 \\
\Delta \sigma / \sigma \leq 0.1
\end{array} \\
\hline \varepsilon_{r, \text { low }}, \sigma_{\text {low }} & 1,1.769 \cdot 10^{-6} \mathrm{~S} / \mathrm{m} \\
\varepsilon_{r, \text { up }}, \sigma_{\text {up }} & 84.458,1.573 \cdot 10^{-3} \mathrm{~S} / \mathrm{m}
\end{array}
$$

Table 2. Concretes: (a) comparing domains of $\sigma$, foreseen by Sensitivity approach and Transfer method, once selected $\varepsilon_{r, \text { concrete }}$ and designed optimally $x_{W, \text { concrete }}$; (b) domains of $\varepsilon$ and $\sigma$ where $\Delta \varepsilon_{r} / \varepsilon_{r} \leq 0.1$ and $\Delta \sigma / \sigma \leq 0.1$, once designed optimally $x_{W, \text { concrete. }}$.

within which the inaccuracy $\Delta \varepsilon_{r} / \varepsilon_{r}\left(L, \sigma, \varepsilon_{r}\right)$ for $\varepsilon_{r}$ is below about 10\% (Figure 11);

having fixed the frequency band, the probe in capacitive contact usually performs optimal measurements over surfaces of lower conductivities compared to when the probe is in galvanic contact, as the respective conductivities are higher by almost one order of magnitude (Tables 1, 3).

\section{Quadrupole configurations}

The transfer impedance of a quadrupolar array can be evaluated for any arbitrary configuration. As a general rule, it is assumed that subsurface electrical sounding becomes scarcely effective at depths greater than the horizontal distance between the electrodes [Grard and Tabbagh 1991, Vannaroni et al. 2004]. This study considers two kinds of probes, i.e. with linear Wenner and square configurations. The linear Wenner arrangement consists of four terminals equally spaced from one another along a straight horizontal line [Vannaroni et al. 2004]. Instead, the square configuration is an array of two parallel horizontal dipoles, with the four electrodes positioned at the corners of a square [Grard and Tabbagh 1991].

If the quadrupole probe (Figure 1) has a characteristic geometrical dimension $L$, then the linear Wenner configuration (Figure 2a) measures a capacitance in a vacuum $C_{0, W}=4 \pi \varepsilon_{0} \cdot L$, while in the square arrangement (Figure 2b) $C_{0, S}=\alpha \cdot C_{0, W}$, is greater by a factor $\alpha=1 /\left(2-2^{1 / 2}\right)>1$.

When the quadrupole is in galvanic contact, i.e. $h=0$, with a subjacent medium of electrical conductivity $\sigma$ and dielectric permittivity $\varepsilon_{r}$, the linear Wenner configuration measures a resistance $R_{N, W}=2 \varepsilon_{0} / \sigma C_{0, W}$ and a parallel capacitance $C_{N, W}=C_{0, W}\left(\varepsilon_{r}+1\right) / 2$, while in the square arrangement, $R_{N, S}=R_{N, W} / \alpha$ and $C_{N, S}=\alpha \cdot C_{N, W}$. So, at the frequency $f$, the transfer impedance $1 / Z_{N}=1 / R_{N}+j 2 \pi f C_{N}$ for the linear Wenner configuration is defined by a modulus $|Z|_{N, W}=1 /\left[\left(1 / R_{N, W}\right)^{2}+\left(2 \pi f C_{N, W}\right)\right]^{1 / 2}$ and a phase $\Phi_{N, W}=\operatorname{arctg}$ $\left(2 \pi f \cdot R_{N, W} C_{N, W}\right)$, while in the square arrangement, $|Z|_{N, S}=$ $\left.Z\right|_{N, W} / \alpha$, which is smaller by a factor of $1 / \alpha$ (Figure $9 a$ ), and $\Phi_{N, S}=\Phi_{N, W}$, which is maintained invariant in the linear Wenner or square configurations (Figure $9 \mathrm{~b}$ ). The cut-off frequency is also independent of the configurations, i.e. $f_{T}=f_{T}\left(\sigma, \varepsilon_{r}\right)$.

Moreover, if the probe grazes the medium and considering that the sensitivity functions are defined as normalized functions, then the sensitivities $S_{\sigma}^{|Z|}$ and $S_{\sigma}^{\Phi_{z}}$ relative to the conductivity $\sigma$, and the functions $S_{\varepsilon_{r}}^{|Z|}\left(\varepsilon_{r}\right)$ and $S_{\varepsilon_{r}}^{\Phi_{z}}\left(\varepsilon_{r}\right)$ relative to the permittivity $\varepsilon_{r}$, for the transfer impedance, both in modulus $|Z|$ and in phase $\Phi_{Z}$, are invariant in the linear Wenner or square configurations. Only if $h=0$ are the inaccuracies $\Delta \sigma / \sigma$ in the measurement of $\sigma$ and $\Delta \varepsilon_{r} / \varepsilon_{r}$ for $\varepsilon_{r}$ also independent of the configurations, so the probe is characterized by the same performances in the frequency band $B$ and in the measurable ranges of $\sigma$ and $\varepsilon_{r}$ (Figure 9c).

Instead, when the quadrupole is in capacitive contact with the subjacent medium, and so the ratio $x=h / L$ between its height $h$ above ground and its electrodeelectrode distance $L$ is not zero, i.e. $0<x \leq 1$, then the quadrupole is characterized by a geometrical factor $K(x)$ $[\delta(x)]$, decreasing (or increasing) the function of $x$, which in the square configuration slopes down (or up) more steeply than in the linear Wenner arrangement, so assuming smaller (or larger) values especially for $1 / 2<\mathrm{x}<1$ (Figure $8 \mathrm{a}$ ). As a consequence, a probe with a fixed $L$ that performs measurements on a medium of dielectric permittivity $\varepsilon_{r}$ could be designed with an optimal height/dimension ratio $x_{\text {opt }}=h_{\text {opt }} / L$, which in the square configuration is smaller than in the linear Wenner arrangement, because its factor $\delta(x)$ slopes up more steeply, increasing the ratio $x$, so reaching the prefixed optimal value $\delta_{\text {opt }}\left(\varepsilon_{r}\right) \approx 2 /\left(15 \varepsilon_{r}+17\right)$ with a smaller $x_{\text {opt }}$. In simpler terms, if the probe is in capacitive contact with the medium, to perform optimal measurements of the permittivity, the square configuration needs to be raised above ground by less than in the linear Wenner arrangement, if their electrode-electrode distances are equal. Indeed, $x$ ranges from $x_{W, \text { low }}=0.022$ in the linear Wenner configuration, and from $x_{S, l o w}=0.019$ in the square arrangement.

Moreover, in the case of capacitive contact, if the quadrupole with electrode-electrode distance $L$ is designed according to the optimal height/dimension ratio $x_{\text {opt }}=h_{\text {opt }} / L$ working in a frequency $f$, then the transfer impedance $Z\left(f, x_{\text {opt }}\right)$, in units of $1 / h_{\text {opt }}$, calculated in $x_{\text {opt }}$, is defined by a phase $\Phi\left(f, x_{\text {opt }}\right)$, which does not depend on the square or linear Wenner configurations (Figure $8 \mathrm{~d}$ ), and by a modulus $|Z|\left(f, x_{o p t}\right)$, in units of $1 / h_{\text {opt }}$, which in the square configuration is shifted down by a factor $1 / \alpha$ with respect to the linear Wenner configuration (Figure $8 \mathrm{c}$ ), remaining 


\begin{tabular}{cc}
$h=0$ & $\begin{array}{c}\Delta \varepsilon_{r} / \varepsilon_{r} \leq 0.1 \\
\Delta \sigma / \sigma \leq 0.1\end{array}$ \\
\hline$\varepsilon_{r, \text { low }}, \sigma_{\text {low }}$ & $1,5.333 \cdot 10^{-5} \mathrm{~S} / \mathrm{m}$ \\
$\varepsilon_{r, \text { up }}, \sigma_{\text {up }}$ & $81,3.14 \cdot 10^{-3} \mathrm{~S} / \mathrm{m}$
\end{tabular}

Table 3. Galvanic contact: domains of $\varepsilon$ and $\sigma$ where $\Delta \varepsilon_{r} / \varepsilon_{r} \leq 0.1$ and $\Delta \sigma / \sigma \leq 0.1$, when $h=0$.

almost unvaried in both configurations not only the shape of the modulus $|Z|\left(f, x_{\text {opt }}\right)$, but also the position of its zero $z\left(x_{\text {opt }}\right)$ and pole $p\left(x_{\text {opt }}\right)$ frequencies (Figure $8 \mathrm{~b}$ ).

Finally, the inaccuracies $\Delta \sigma / \sigma\left(f, x_{\text {opt }}\right)$ in the measurements of the conductivity $\sigma$ and $\Delta \varepsilon_{r} / \varepsilon_{r}\left(f, x_{\text {opt }}\right)$ for the permittivity $\varepsilon_{r}$, calculated in $x_{o p t}$, do not depend on the two configurations, so the optimal frequency $f_{\text {opt }}\left(x_{\text {opt }}\right)$ that minimizes the inaccuracy $\Delta \varepsilon_{r} / \varepsilon_{r}\left(f, x_{o p t}\right)$ for $\varepsilon_{r}$, together with the minimum and maximum values of frequency $f_{\min }\left(x_{\text {opt }}\right)$ and $f_{\max }\left(x_{\text {opt }}\right)$, respectively, which allow the inaccuracy $\Delta \varepsilon_{r} / \varepsilon_{r}$ $\left(f, x_{\text {opt }}\right)$ below a prefixed limit $(10 \%)$, are invariant in both of the configurations (Figure 8e). In simpler terms, if the probe is in capacitive contact with the medium, to perform an optimal measurement of permittivity considering different height/dimension ratios, the design of the two configurations establishes (almost) invariant trends in frequency, both for their transfer impedances and measurement inaccuracies.

\section{Conclusions}

The present study has proposed a theoretical modeling of simultaneous and noninvasive measurements of electrical resistivity and dielectric permittivity using a quadrupole probe on a subjacent medium [see also arXiv.org's ref.: Settimi et al. 2009]. A mathematical-physical model has been applied to the propagation of errors in the measurement of resistivity and permittivity based on the sensitivity functions tool. The findings have also been compared to the results of the classical method of analysis in the frequency domain, which is useful for determining the behaviour of zero and pole frequencies in the linear time invariant circuit of the quadrupole. This study has underlined that average values of electrical resistivity and dielectric permittivity can be used to estimate the complex impedance over various terrains and concretes, especially when they are characterized by low levels of water saturation or content [Knight and Nur 1987], and are analyzed within a bandwidth ranging from only LFs to MFs [Al-Qadi et al. 1995, Myounghak et al. 2007]. To meet the design specifications that ensure satisfactory performances of the probe (inaccuracy of no more than $10 \%)$, the forecasts provided by the theory of error propagation suggested by Vannaroni et al. [2004] that apply the sensitivity functions approach, as explicitly developed in the study, have been discussed in comparison to those foreseen by the analysis in the frequency domain suggested by Grard and Tabbagh [1991]. Here, this deepens the transfer function method to analyze the zero and pole behavior (in terms of both band of frequency $f$ and measurable range of resistivity $\rho$, or permittivity $\varepsilon_{r}$ ).

It is interesting to compare the results of the present study with others in the literature [Grard and Tabbagh 1991,

\section{Domain $\left(\varepsilon_{\mathrm{r}}, \sigma\right)$ for $\Delta \varepsilon_{\mathrm{r}} / \varepsilon_{\mathrm{r}} \leq 0.1$ and $\Delta \sigma / \sigma \leq 0.1$}

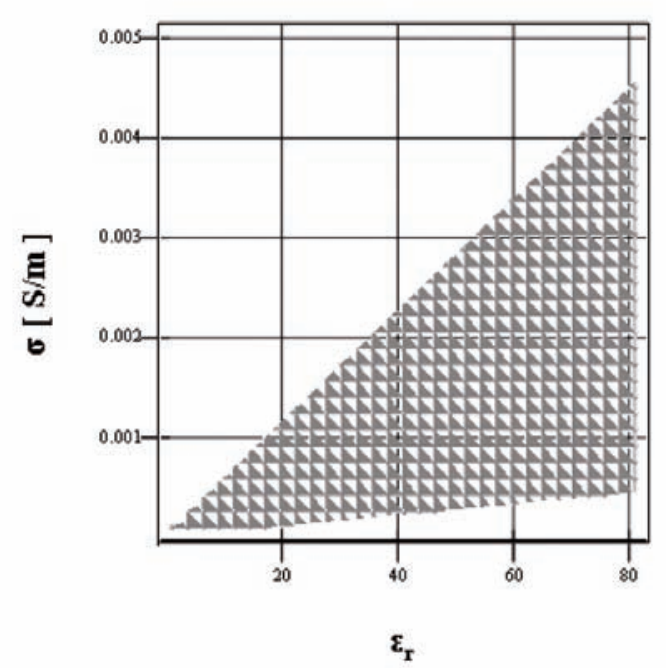

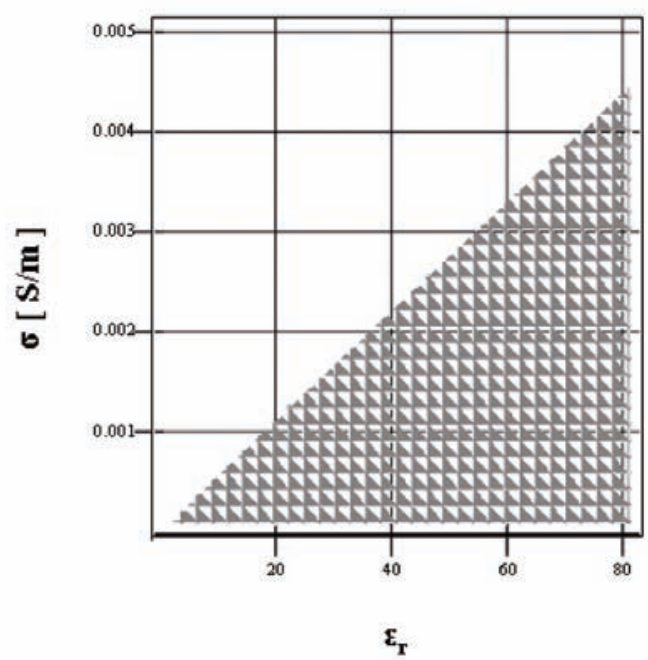

(b)

Figure 11. With reference to a quadrupole probe in galvanic contact, working in a fixed band $B=100 \mathrm{kHz}$, plots for the domains $\left(\sigma, \varepsilon_{r}\right)$ of the electrical conductivity $\sigma$ and the dielectric permittivity $\varepsilon_{r}$ such that: (a) the transfer impedance is characterized by a modulus with a cut-off frequency $f_{T}=f_{T}\left(\sigma, \varepsilon_{r}\right)=\sigma /\left(2 \pi \varepsilon_{0}\left(\varepsilon_{r}+1\right)\right)$ in the interval $f_{T} \in[100 \mathrm{kHz}, 1 \mathrm{MHz}]$; (b) both the inaccuracies $\Delta \sigma / \sigma\left(\sigma, \varepsilon_{r}\right)$ in the measurement of the conductivity $\sigma$, and $\Delta \varepsilon_{r} / \varepsilon_{r}\left(\sigma, \varepsilon_{r}\right)$ of the permittivity $\varepsilon_{r}$ are below a prefixed limit of $10 \%\left[\Delta|Z| /|Z|=\Delta \Phi_{Z} / \Phi_{Z}=10^{-3}\right]$. See also Tables 1 and 3 . 
Vannaroni et al. 2004]. In agreement, the sensitivity functions approach provides the following results: a) if the quadrupole probe is in galvanic contact with the subsurface, i.e. $h=0$, then the inaccuracies $\Delta \sigma / \sigma$ in the measurement of conductivity $\sigma$ and $\Delta \varepsilon_{r} / \varepsilon_{r}$ for permittivity $\varepsilon_{r}$ are minimized in the frequency band $B$ of the quadrupole for all of its geometrical configurations and media; and $b$ ) even if $h \neq 0$, the design of the probe must be optimized with reference to the minimum value of the inaccuracy $\Delta \varepsilon_{r} / \varepsilon_{r}$ for $\varepsilon_{r}$, which is always higher than the corresponding minimum value of the inaccuracy $\Delta \sigma / \sigma$ in the band $B$ for all its configurations and media.

More explicitly than in these previous studies [Grard and Tabbagh 1991,Vannaroni et al. 2004], the transfer functions method provides results such that to satisfy the operative conditions of linearity for the measurements: a) if the quadrupole has capacitive contact with the subjacent medium, then the frequency $f$ of the probe should be imposed as included between the zero $z_{M}$ and the pole $p_{M}$ of the transfer impedance, and so its modulus is almost constant within the frequency band. An optimization equation is deduced for the probe that links the optimal ratio $x$ between its height above ground and its characteristic geometrical dimension only to the dielectric permittivity $\varepsilon_{r}$ of the medium; b) instead, if the quadrupole is in galvanic contact with the subjacent medium, then the working frequency $f$ of the quadrupole should be imposed as lower than the cut-off frequency of the transfer impedance, and so its modulus is constant below the cut-off frequency. It is optimal to design the characteristic geometrical dimensions of the probe or to establish the measurable ranges of the conductivity $\sigma$ and permittivity $\varepsilon_{r}$ of the medium.

Unlike these previous studies [Grard and Tabbagh 1991, Vannaroni et al. 2004], the sensitivity functions approach and the transfer functions method provide results that allow an assessment of the performance of the quadrupole probe in galvanic and capacitive contact: a) on a selected surface (for example, a non-saturated concrete with low conductivity $\sigma=10^{-4} \mathrm{~S} / \mathrm{m}$ and $\varepsilon_{r}=4$ ), it is usually possible to verify that the quadrupole in capacitive contact performs optimal measurements over the band $\left[f_{\min }\left(x_{\text {opt }}\right)<z\left(x_{\text {opt }}\right), f_{\text {max }}\left(x_{\text {opt }}\right)>p\left(x_{\text {opt }}\right)\right]$, which is shifted to lower and higher frequencies compared to when the probe is in galvanic contact, as the respective band $\left[f_{\min }, f_{\max }\right]$ is narrower by almost one LF-MF decade in frequency, particurarly increasing the value of $\varepsilon_{r}$; b) having fixed the frequency band, the quadrupole in capacitive contact usually performs optimal measurements over surfaces of lower conductivity compared to when the probe is in galvanic contact, as the respective conductivities are higher by almost one order of magnitude.

On this basis, some constraints were established to design a quadrupole probe for conducting measurements of electrical resistivity and dielectric permittivity in a regime of $\mathrm{AC}$ at LFs and MFs $(10 \mathrm{kHz}-1 \mathrm{MHz})$. Measurements were carried out using four electrodes laid on the surface to be analyzed, and through measurement of transfer impedance, the resistivity and permittivity of the material can be extracted. Furthermore, by increasing the distance between the electrodes, the electrical properties of the sub-surface structures can be investigated to greater depths. The main advantage of the quadrupole is that measurements of electrical parameters can be conducted with a nondestructive technique, thereby enabling characterization of precious and unique materials. Also, in appropriate arrangements, measurements could be carried out with electrodes slightly raised above the surface, allowing for completely nondestructive analysis, although accompanied by a greater error. The probe can perform measurements on materials with high resistivity and permittivity in an immediate way, without the need for later stages of data post-analysis.

\section{References}

Al-Qadi, I. L., O. A. Hazim, W. Su and S. M. Riad (1995). Dielectric properties of Portland cement concrete at low radio frequencies, J. Mater. Civil. Eng., 7, 192-198.

Auty, R. P. and R. H. Cole (1952). Dielectric properties of ice and solid, J. Chem. Phys., 20, 1309-1314.

Banton, O., M. K. Seguin and M. A. Cimon (1997). Mapping field scale physical properties of soil with electrical resistivity, Soil Sci. Soc. Am. J., 61, 1010-1017.

Chelidze, T.L. and Y. Gueguen (1999). Electrical spectroscopy of porous rocks: a review - I, Theoretical models, Geophys. J. Int., 137, 1-15.

Chelidze, T.L., Y. Gueguen and C. Ruffet (1999). Electrical spectroscopy of porous rocks: a review - II, Experimental results and interpretation, Geophys. J. Int., 137, 16-34.

Debye, P. (1929). Polar Molecules, Leipzig.

Del Vento, D. and G. Vannaroni (2005). Evaluation of a mutual impedance probe to search for water ice in the Martian shallow subsoil, Rev. Sci. Instrum., 76, 084504 (1-8).

Edwards, R. J. (1998). Typical Soil Characteristics of Various Terrains, http:/ / www.smeter.net/grounds/ soil-electricalresistance.php.

Fechant, C. (1996). Réalisation d'un quadripôle de mesure in situ de la permitivié diélectrique des végétaux. Premier application à la détermination du contenu en eau des épis de blé, These de l'Université Pierre-et-Marie-Curie VI, Paris.

Fechant, C. and A. Tabbagh (1999). Mesure en laboratoire de la permittivité diélectrique moyenne fréquence de végétaux à $430 \mathrm{kHz}$ à l'aide d'un capacimétre. Relation entr permittivité apparente d'un ensemble d'épis de blé et leur contenu en eau, C. R. Acad. Sci. Paris t. 327 Série II b, 285-298 (both in French and in English).

Frölich, H. (1990 edition). Theory of Dielectrics, Oxford. Grard, R. (1990a). A quadrupolar array for measuring the 
complex permittivity of the ground: application to earth prospection and planetary exploration, Meas. Sci. Technol., 1, 295-301.

Grard, R. (1990b). A quadrupole system for measuring in situ the complex permittvity of materials: application to penetrators and landers for planetary exploration, Meas. Sci. Technol., 1, 801-806.

Grard, R. and A. Tabbagh (1991). A mobile four electrode array and its application to the electrical survey of planetary grounds at shallow depth, J. Geophys. Res., 96, 4117-4123.

Kearey, P., M. Brooks and I. Hill (2002). An introduction to geophysical exploration, Oxford.

Knight, R. J. and A. Nur (1987). The dielectric constant of sandstone, $60 \mathrm{kHz}$ to $4 \mathrm{MHz}$, Geophysics, 52, 644-654.

Laurents, S., J. P. Balayssac, J. Rhazi, G. Klysz and G. Arliguie (2005). Non-destructive evaluation of concrete moisture by GPR: experimental study and direct modeling, Materials and Structures (M\&S), 38, 827-832.

Loke, M. H. (2001). Tutorial: 2-D and 3-D electrical imaging surveys, Course Notes for USGS Workshop 2-D and 3-D Inversion and Modelling of Surface and Borehole Resistivity Data, Torrs, CT.

Murray-Smith, D. J. (1987). Investigations of methods for the direct assessment of parameter sensitivity in linear closed-loop control systems, in Complex and distributed systems: analysis, simulation and control, edited by S. G. Tzafestas and P. Borne, Amsterdam, 323-328.

Myounghak, O., K. Yongsung and P. Junboum (2007). Factors affecting the complex permittivity spectrum of soil at a low frequency range of $1 \mathrm{kHz} 10 \mathrm{MHz}$, Environ Geol., 51, 821-833.

Polder, R., C. Andrade, B. Elsener, Ø. Vennesland, J. Gulikers, R. Weidert and M. Raupach (2000). Test methods for on site measurements of resistivity of concretes, Materials and Structures (M\&S), 33, 603-611.

Rhoades, J. D., P. A. C. Raats and R. J. Prather (1976). Effect of liquid-phase electrical conductivity, water content, and surface conductivity on bulk soil electrical conductivity, Soil Sci. Soc. Am. J., 40, 651-655.

Samouëlian, A., I. Cousin, A. Tabbagh, A. Bruand and G. Richard (2005). Electrical resistivity survey in soil science: a review, Soil Till. Res., 83, 172-193.

Settimi, A., A. Zirizzotti, J. A. Baskaradas and C. Bianchi (2009). Inaccuracy assessment for simultaneous measurements of resistivity and permittivity applying sensitivity and transfer function approaches, arXiv.org: 0908.0641.

Tabbagh, A. (1994). Simultaneous measurement of electrical and dielectric permittivity of electrical conductivity and dielectric permittivity of soil using a slingram electromagnetic device in medium frequency range, Archaeometry, 36, 159-170.

Tabbagh, A., A. Hesse and R. Grard (1993). Determination of electrical properties of the ground at shallow depth with an electrostatic quadrupole: field trials on archaeological sites, Geophys. Prospect., 41, 579-597.

Vannaroni, G., E. Pettinelli, C. Ottonello, A. Cereti, G. Della Monica, D. Del Vento, A. M. Di Lellis, R. Di Maio, R. Filippini, A. Galli, A. Menghini, R. Orosei, S. Orsini, S. Pagnan, F. Paolucci, A. Pisani R., G. Schettini, M. Storini and G. Tacconi (2004). MUSES: multi-sensor soil electromagnetic sounding, Planet. Space Sci., 52, 67-78.

Wenner, F. (1915). A method of measuring earth resistivity, US Bur. of Stand. Bull., 12, 469-478. 


\section{Appendix A}

We consider here the influence of inaccuracies in transfer impedance in modulus and phase on the measurement of electrical conductivity and dielectric permittivity. The mathematical tool best suited to this purpose applies the so-called sensitivity functions [MurraySmith 1987], which formalize the intuitive concept of sensitivity as the ratio between the percentage error of certain physical quantities (due to the variation of some parameters) and the percentage error of the same parameters.

The inaccuracies $\Delta \sigma / \sigma$ in the measurement of the electrical conductivity $\sigma$, and $\Delta \varepsilon_{r} / \varepsilon_{r}$ for the dielectric permittivity $\varepsilon_{r}$, can be expressed as linear combinations of the inaccuracies $\Delta|Z| /|Z|$ and $\Delta \Phi_{Z} / \Phi_{Z}$ in the measurement of transfer impedance in modulus $|Z|$ and in phase $\Phi_{Z}$, respectively, as given in Equations (4.1) and (4.2) (Figure 3). The pairs of sensitivity functions $\left(S_{\sigma}^{|Z|}, S_{\sigma}^{\Phi_{z}}\right)$ and $\left(S_{\varepsilon_{r}}^{|Z|}, S_{\varepsilon_{r}}^{\Phi_{z}}\right)$ for the transfer impedance, both in $|Z|$ and $\Phi_{Z}$, relative to the conductivity $\sigma$ and the permittivity $\varepsilon_{r}$ (Figure 4),

$$
\begin{aligned}
S_{\sigma}^{|Z|} & =\frac{\Delta|Z| /|Z|}{\Delta \sigma / \sigma}=\frac{\partial|Z|}{\partial \sigma} \frac{\sigma}{|Z|}= \\
& =\frac{1}{S_{|Z|}^{\sigma}}=\frac{1}{2} H_{1} S_{\sigma}^{R}-\frac{1}{2} H_{2} S_{\sigma}^{C}, \text { for } \varepsilon_{r}=\text { const },
\end{aligned}
$$

$$
\begin{aligned}
& S_{\sigma}^{\Phi_{z}}=\frac{\partial \Phi_{Z}}{\partial \sigma} \frac{\sigma}{\Phi_{Z}}=\frac{1}{S_{\Phi_{z}}^{\sigma}}=\frac{1}{2} \frac{\Omega \frac{R}{R_{N}} \frac{C}{C_{N}}}{\operatorname{arct}\left(\Omega \frac{R}{R_{N}} \frac{C}{C_{N}}\right)} H_{1}\left(S_{\sigma}^{R}+S_{\sigma}^{C}\right) \cong \\
& \cong \frac{1}{2} H_{1}\left(S_{\sigma}^{R}+S_{\sigma}^{C}\right), \text { for } \Omega \frac{R}{R_{N}} \frac{C}{C_{N}} \leq 1\left(\varepsilon_{r}=\text { const }\right)
\end{aligned}
$$

$S_{\mathcal{E}_{r}}^{|Z|}=\frac{1}{S_{|Z|}^{\mathcal{E}_{r}}}=\frac{1}{2} H_{1} S_{\mathcal{E}_{r}}^{R}-\frac{1}{2} H_{2} S_{\varepsilon_{r}}^{C}$, for $\sigma=$ const,

$$
\begin{aligned}
& S_{\varepsilon_{r}}^{\Phi_{z}}=\frac{1}{S_{\Phi_{z}}^{\varepsilon_{r}}}=\frac{1}{2} \frac{\Omega \frac{R}{R_{N}} \frac{C}{C_{N}}}{\operatorname{arct}\left(\Omega \frac{R}{R_{N}} \frac{C}{C_{N}}\right)} H_{1}\left(S_{\varepsilon_{r}}^{R}+S_{\varepsilon_{r}}^{C}\right) \cong \\
& \cong \frac{1}{2} H_{1}\left(S_{\varepsilon_{r}}^{R}+S_{\varepsilon_{r}}^{C}\right), \text { for } \Omega \frac{R}{R_{N}} \frac{C}{C_{N}} \leq 1(\sigma=\text { const }),
\end{aligned}
$$

are, in turn, linear combinations of the sensitivity function pairs $\left(S_{\sigma}^{R}, S_{\sigma}^{C}\right)$ and $\left(S_{\varepsilon^{2}}^{R}, S_{\varepsilon}^{C}\right)$ for transfer impedance, in both the resistance $R$ and capacitance $C$ parallel components, relative to $\sigma$ and $\varepsilon_{r}$,

$$
S_{\sigma}^{R}=\left.\frac{\Delta R / R}{\Delta \sigma / \sigma}\right|_{\varepsilon_{r}=\text { const }}=\left.\frac{\partial R}{\partial \sigma}\right|_{\varepsilon_{r}=\text { const }} \frac{\sigma}{R}=
$$

$$
=-\frac{\left[1+\delta(x) \frac{\varepsilon_{r}-1}{2}\right]^{2}-\left[\frac{\delta(x)}{\Omega} \frac{\varepsilon_{r}+1}{2}\right]^{2}}{\left[1+\delta(x) \frac{\varepsilon_{r}-1}{2}\right]^{2}+\left[\frac{\delta(x)}{\Omega} \frac{\varepsilon_{r}+1}{2}\right]^{2}},
$$

$$
\begin{aligned}
& S_{\sigma}^{C}=\left.\frac{\partial C}{\partial \sigma}\right|_{\mathcal{E}_{r}=\text { const }} \frac{\sigma}{C}= \\
& \begin{array}{r}
\frac{1}{2} \frac{\varepsilon_{r}+1}{\Omega^{2}} \frac{\delta(x)\left[2+\delta(x)\left(\varepsilon_{r}-3\right)-\delta^{2}(x)\left(\varepsilon_{r}-1\right)\right]}{\left[1+\delta(x)\left(\frac{\varepsilon_{r}-1}{2}+\frac{1}{\Omega^{2}} \frac{\varepsilon_{r}+1}{2}\right)\right][(1+} \\
\left.\left.\quad+\delta(x) \frac{\varepsilon_{r}-1}{2}\right)^{2}+\left(\frac{\delta(x)}{\Omega} \frac{\varepsilon_{r}+1}{2}\right)^{2}\right]
\end{array} \\
& \left.S_{\varepsilon_{r}}^{R}\right|_{\sigma=\text { const }}=\varepsilon_{r} \frac{\delta(x)\left[1+\delta(x) \frac{\varepsilon_{r}-1}{2}\right]}{\left[1+\delta(x) \frac{\varepsilon_{r}-1}{2}\right]^{2}+\left[\frac{\delta(x)}{\Omega} \frac{\varepsilon_{r}+1}{2}\right]^{2}},
\end{aligned}
$$

$$
\begin{aligned}
&\left.S_{\varepsilon_{i}}^{C}\right|_{\sigma=\text { const }}= \\
& 1+\delta(x)\left(\varepsilon_{r}-2\right)+\frac{1}{4} \delta^{2}(x)\left[\left(\varepsilon_{r}-1\right)\left(\varepsilon_{r}-5\right)-\right. \\
&=\frac{\varepsilon_{r}}{\varepsilon_{r}+1} \frac{\left.-\left(\frac{\varepsilon_{r}+1}{\Omega}\right)^{2}\right]-\frac{1}{4} \delta^{3}(x)\left[\left(\varepsilon_{r}-1\right)^{2}-\left(\frac{\varepsilon_{r}+1}{\Omega}\right)^{2}\right]}{\left[1+\delta(x)\left(\frac{\varepsilon_{r}-1}{2}+\frac{1}{\Omega^{2}} \frac{\varepsilon_{r}+1}{2}\right)\right][(1+}, \\
&\left.\left.+\delta(x) \frac{\varepsilon_{r}-1}{2}\right)^{2}+\left(\frac{\delta(x)}{\Omega} \frac{\varepsilon_{r}+1}{2}\right)^{2}\right]
\end{aligned}
$$

with the weight functions:

$$
\begin{aligned}
H_{1}= & \frac{[1-\delta(x)]^{2}}{1+\Omega^{2}+\delta(x)\left(\varepsilon_{r}-1\right)\left(1+\Omega^{2}\right)+}, \\
& +\delta^{2}(x)\left(1+\Omega \frac{\varepsilon_{r}-1}{2}+\frac{1}{\Omega} \frac{\varepsilon_{r}+1}{2}\right)^{2}
\end{aligned}
$$

$$
\begin{aligned}
\Omega^{2}+2 \delta(x)\left(\Omega^{2} \frac{\varepsilon_{r}-1}{2}+\frac{\varepsilon_{r}+1}{2}\right)+ \\
H_{2}=\frac{+\delta^{2}(x)\left(\Omega \frac{\varepsilon_{r}-1}{2}+\frac{1}{\Omega} \frac{\varepsilon_{r}+1}{2}\right)^{2}}{1+\Omega^{2}+\delta(x)\left(\varepsilon_{r}-1\right)\left(1+\Omega^{2}\right)+} . \\
+\delta^{2}(x)\left(1+\Omega \frac{\varepsilon_{r}-1}{2}+\frac{1}{\Omega} \frac{\varepsilon_{r}+1}{2}\right)^{2}
\end{aligned}
$$

Considering Equations (A.1) to (A.4), if the modulus $|Z|$ and the phase $\Phi_{Z}$ of the transfer impedance provide an indirect measurement of the electrical conductivity $\sigma$ and dielectric permittivity $\varepsilon_{r}$, then the functions $|Z|=|Z|\left(\sigma, \varepsilon_{r}\right)$ and $\Phi_{Z}=\Phi_{Z}\left(\sigma, \varepsilon_{r}\right)$ are invertible, i.e. $\sigma=\sigma\left(|Z|, \Phi_{Z}\right)$ and $\varepsilon_{r}=$ $\varepsilon_{r}\left(|Z|, \Phi_{Z}\right)$. Therefore, the theorem of the derivative for the 
inverse function can be applied. Indeed, under the condition $\sigma=$ const (or $\varepsilon_{r}=$ const), both $|Z|$ and $\Phi_{Z}$ are invertible functions of $\varepsilon_{r}$ (or $\sigma$ ), i.e. they are strictly increasing or decreasing monotonic functions of $\varepsilon_{r}($ or $\sigma)$.

\section{Appendix B}

By exact calculations, the transfer impedance $Z$ (f, $x, \sigma$, $\varepsilon_{r}$ ) measured by the quadrupole probe, in units of the reciprocal height $1 / h$ from the subjacent medium, consists of the resistance $R\left(f, x, \sigma, \varepsilon_{r}\right)$, in units of $1 / h$ - see Equation (3.10) -, which can be expressed as a transfer function characterized by a pole in the origin frequency $p_{R}=0$, a zero in higher frequencies $z_{R}\left(f, x, \sigma, \varepsilon_{r}\right)>0$, and a static gain $K_{R}(f$, $x, \sigma)$,

$R\left(f, x, \sigma, \varepsilon_{r}\right)=K_{R}(x, \sigma) \frac{1+\frac{f^{2}}{z_{R}^{2}\left(x, \sigma, \varepsilon_{r}\right)}}{(2 \pi f)^{2}}$,

where:

$z_{R}\left(x, \sigma, \varepsilon_{r}\right)=\frac{1}{2 \pi} \frac{\sigma}{\varepsilon_{0}\left(\varepsilon_{r}+1\right)} \frac{\delta(x) \frac{\varepsilon_{r}+1}{2}}{1+\delta(x) \frac{\varepsilon_{r}-1}{2}}$,

$K_{R}(x, \sigma)=\frac{1}{2} \frac{\sigma}{\varepsilon_{0}} \frac{1}{C_{0}(x)} \frac{\delta^{2}(x)}{1-\delta(x)}$,

as well as the parallel capacitance $C\left(f, x, \sigma, \varepsilon_{r}\right)$, in units of $1 / h$ - see Equation (3.11) -, which can be expressed as a transfer function characterized by a low frequency pole $p_{C}(f$, $\left.x, \sigma, \varepsilon_{r}\right)$, a zero in higher frequencies $z_{C}\left(f, x, \sigma, \varepsilon_{r}\right)>p_{C}(f, x$, $\left.\sigma, \varepsilon_{r}\right)$, and a static gain $K_{C}(x)$,

$C\left(f, x, \sigma, \varepsilon_{r}\right)=K_{C}(x) \frac{1+\frac{f^{2}}{z_{C}^{2}\left(x, \sigma, \varepsilon_{r}\right)}}{1+\frac{f^{2}}{p_{C}^{2}\left(x, \sigma, \varepsilon_{r}\right)}}$,

where the capacitance pole $p_{C}\left(f, x, \sigma, \varepsilon_{r}\right)$ coincides with the resistance pole $z_{R}\left(f, x, \sigma, \varepsilon_{r}\right)$,

$$
p_{C}\left(x, \sigma, \varepsilon_{r}\right)=z_{C}\left(x, \sigma, \varepsilon_{r}\right),
$$

and:

$$
z_{C}\left(x, \sigma, \varepsilon_{r}\right)=\frac{1}{2 \pi} \frac{\sigma}{\varepsilon_{0}\left(\varepsilon_{r}+1\right)} \sqrt{\frac{\delta(x) \frac{\varepsilon_{r}+1}{2}}{1+\delta(x) \frac{\varepsilon_{r}-1}{2}}},
$$

$$
K_{C}(x)=\frac{C_{0}(x)}{\delta(x)} .
$$

For values of the ratio $x=h / L$ between the height $h$ above ground and the characteristic geometrical dimension $L$, and of the paired values of electrical conductivity $\sigma$ and dielectric permittivity $\varepsilon_{r}$ that satisfy the condition (Figure 5),

$$
\frac{1}{\left[K_{R}(x, \sigma) K_{C}(x)\right]^{2}}<<\frac{2}{\left[2 \pi \cdot z_{C}\left(x, \sigma, \varepsilon_{r}\right)\right]^{2}},
$$

it can be demonstrated that the modulus $|Z|\left(f, x, \sigma, \varepsilon_{r}\right)$ can be approximately expressed as a transfer function with a pole in the origin frequency, a low frequency zero $z_{M}\left(f, x, \sigma, \varepsilon_{r}\right)$, a pole in higher frequencies $p_{M}\left(f, x, \sigma, \varepsilon_{r}\right)>z_{M}\left(f, x, \sigma, \varepsilon_{r}\right)$, and a static gain $K_{M}(x)$ (Figure $8 c$ ),

$$
|Z|\left(f, x, \sigma, \varepsilon_{r}\right)=K_{M}(x) \frac{1+\frac{f^{2}}{z_{M}^{2}\left(x, \sigma, \varepsilon_{r}\right)}}{2 \pi f \cdot\left[1+\frac{f^{2}}{p_{M}^{2}\left(x, \sigma, \varepsilon_{r}\right)}\right]},
$$

where the zero of the modulus $z_{M}\left(f, x, \sigma, \varepsilon_{r}\right)$ coincides with the capacitance pole $p_{C}\left(f, x, \sigma, \varepsilon_{r}\right)$ and the pole of the modulus $p_{M}\left(f, x, \sigma, \varepsilon_{r}\right)$ with the capacitance zero $z_{C}\left(f, x, \sigma, \varepsilon_{r}\right)$ (Figure $8 \mathrm{~b}$ ),

$$
\begin{aligned}
& z_{M}\left(x, \sigma, \varepsilon_{r}\right)=p_{C}\left(x, \sigma, \varepsilon_{r}\right), \\
& p_{M}\left(x, \sigma, \varepsilon_{r}\right)=z_{C}\left(x, \sigma, \varepsilon_{r}\right),
\end{aligned}
$$

and

$$
K_{M}(x)=\frac{1}{K_{C}(x)} .
$$

Equation (B.8) establishes limits on the range for the design specification $x$ of the quadrupole and the measurable range $\left(\sigma, \varepsilon_{r}\right)$ of the media.

To satisfy the operative conditions of linearity for the measurements, the quadrupole probe, which is characterized by the height / dimensions ratio $x=h / L$, should measure the conductivity $\sigma$ and the permittivity $\varepsilon_{r}$ of the subjacent medium when its working frequency $f$ falls within the band included between the zero $z_{M}\left(f, x, \sigma, \varepsilon_{r}\right)$ and the pole $p_{M}(f, x$, $\sigma, \varepsilon_{r}$ ) of the transfer impedance, as reported in Equation (4.3).

Moreover, the quadrupole probe specified by $x=h / L$ should measure $\varepsilon_{r}$, as its geometrical factor $\delta(x)$ is close to Equation (4.4), a necessary condition for $Z\left(f, x, \sigma, \varepsilon_{r}\right)$ to show an almost constant modulus within the band (Equation 4.3), the modulus in the zero (Equation B.10) coinciding with the corresponding one in the pole (Equation B.11),

$$
\begin{gathered}
|Z|_{f=z_{M}}\left(x, \sigma, \varepsilon_{r}\right)=\frac{K_{M}(x)}{\pi z_{M}\left(x, \sigma, \varepsilon_{r}\right)}|Z|_{f=p_{M}}\left(x, \sigma, \varepsilon_{r}\right)= \\
=\frac{1}{2} K_{M}(x) \frac{p_{M}\left(x, \sigma, \varepsilon_{r}\right)}{2 \pi z_{M}^{2}\left(x, \sigma, \varepsilon_{r}\right)},
\end{gathered}
$$


so that the pole (Equation B.11) is almost four-fold greater that the zero (Equation B.10),

$$
p_{M}\left(x, \sigma, \varepsilon_{r}\right) \approx 4 z_{M}\left(x, \sigma, \varepsilon_{r}\right) .
$$

Equation (4.4) can be interpreted as the optimization equation of the quadrupole, so the sizing for the height/dimension ratio $x$ of the probe depends only on the permittivity $\varepsilon_{r}$ of the medium; instead, Equations (4.3) and (B.14) show that the probe can work optimally only in a small band of frequencies.

${ }^{\star}$ Corresponding author. Dr. Alessandro Settimi, Istituto Nazionale di Geofisica e Vulcanologia (INGV), Via di Vigna Murata 605,

I-00143 Rome, Italy; e-mail: alessandro.settimi@ingv.it

C 2010 by the Istituto Nazionale di Geofisica e Vulcanologia. All rights reserved. 